\title{
Wave packet pseudomodes of twisted Toeplitz matrices
}

\author{
Lloyd N. Trefethen and S. J. Chapman
}

June 29, 2003

\begin{abstract}
The pseudospectra of nonsymmetric Toeplitz or circulant matrices with varying coefficients are considered. Such matrices are characterized by a symbol that depends on both position $(x)$ and wave number $(k)$. It is shown that when a certain winding number or twist condition is satisfied, related to Hörmander's commutator condition for partial differential equations, $\varepsilon$-pseudoeigenvectors of such matrices for exponentially small values of $\varepsilon$ exist in the form of localized wave packets. The symbol need not be smooth, just differentiable at a point (or less).
\end{abstract}

\section{Introduction}

For a positive integer $N$, define

$$
s_{j}=2 \sin x_{j}, \quad x_{j}=\frac{2 \pi j}{N}, \quad 1 \leq j \leq N,
$$

and consider the $N \times N$ "Scottish flag matrix" that in the case $N=5$ takes the form

$$
A=\left(\begin{array}{ccccc}
s_{1} & 1 & & & -1 \\
-1 & s_{2} & 1 & & \\
& -1 & s_{3} & 1 & \\
& & -1 & s_{4} & 1 \\
1 & & & -1 & s_{5}
\end{array}\right)
$$

This is an example of what we shall call a twisted Toeplitz matrix: a matrix whose diagonals are either constant or (usually continuously or piecewise continuously in a sense to be made precise) varying.

The diagonal part of $A$ is symmetric, with real eigenvalues $\left\{s_{j}\right\}$, and the off-diagonal part is skew-symmetric, with imaginary eigenvalues $\left\{\mathrm{i} s_{j}\right\}$. Thus both of these pieces of $A$ are normal matrices with well-conditioned eigenvalue problems. ${ }^{1} A$ itself, however, is strongly nonnormal. Figure 1 shows its

\footnotetext{
${ }^{1}$ A matrix $A$ is normal if $A^{*} A=A A^{*}$, where $A^{*}$ is the conjugate transpose, or equivalently, if it has a complete set of orthogonal eigenvectors. If $A$ is normal, its eigenvalues are well-conditioned in the sense that each eigenvalue of any perturbed matrix $A+E$ differs from an eigenvalue of $A$ by at most $\|E\|$, where $\|\cdot\|$ is the matrix 2-norm.
} 


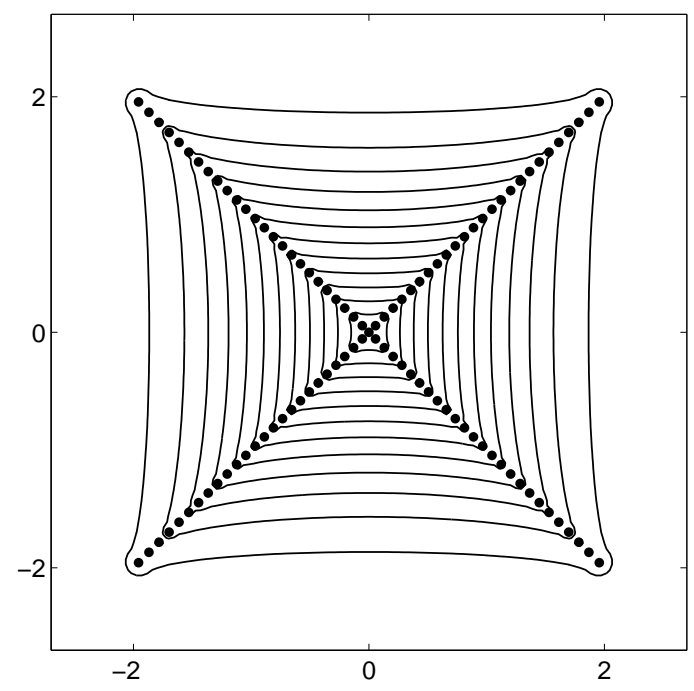

Figure 1: Eigenvalues and $\varepsilon$-pseudospectra of the "Scottish flag matrix" (2) for $N=101$ and $\varepsilon=10^{-1}, 10^{-2}, \ldots, 10^{-12}$. From outside in, we see the level curves $\left\|(\lambda-A)^{-1}\right\|=\varepsilon^{-1}$ for these values of $\varepsilon$.

eigenvalues and 2-norm pseudospectra ${ }^{2}$, computed with the MatLaB package EigTool $[46,47]$, for the case $N=101$. Evidently the eigenvalues are neither real nor imaginary, lying instead on a cross at angle $\pi / 4$ in the square $-2 \leq \operatorname{Re} \lambda, \operatorname{Im} \lambda \leq 2$. But whereas there are only $N$ eigenvalues, the figure reveals that every number $-2<\operatorname{Re} \lambda, \operatorname{Im} \lambda<2$ is an $\varepsilon$-pseudoeigenvalue for a small value of $\varepsilon .^{3}$

Figure 2 shows a pseudospectrum of $A$ in another way by superimposing the eigenvalues of 100 matrices $A+E$, where each $E$ is a random dense complex matrix (independent normally distributed complex entries) of norm $10^{-4}$.

Figure 3 shows one of the wave packet pseudomodes that is the subject of this paper. An $\varepsilon$-pseudoeigenvector or $\varepsilon$-pseudomode of $A$, for given $\varepsilon$ pseudoeigenvalue $\lambda$, is a nonzero vector $v$ such that $\|(A-\lambda) v\| \leq \varepsilon\|v\|$. For this particular vector $v$ and $\lambda=1$, we have

$$
\frac{\|(A-\lambda) v\|}{\|v\|}=5.688 \ldots \times 10^{-6} \approx\left\|(\lambda-A)^{-1}\right\|^{-1} .
$$

The wave packet is centered at $x=x_{*}=\pi-\sin ^{-1}\left(\frac{1}{2}\right) \approx 2.618$ and has wave number centered at $k=k_{*}=\pi$, i.e., a sawtoothed behavior with two points

\footnotetext{
${ }^{2}$ For each $\varepsilon>0$, the $\varepsilon$-pseudospectrum of $A$ is the set of complex numbers $\lambda$ for which $\left\|(\lambda-A)^{-1}\right\| \geq \varepsilon^{-1}$, or equivalently, which are eigenvalues of some matrix $A+E$ with $\|E\| \leq \varepsilon[40,41]$. (Throughout this article we take $\|\cdot\|=\|\cdot\|_{2}$. If $\lambda$ is an eigenvalue of $A$ we define $\left\|(\lambda-A)^{-1}\right\|=\infty$.) Extensive information about pseudospectra, including a list of applications and a bibliography of several hundred papers, is available online at the Pseudospectral Gateway [18].

${ }^{3}$ The spectrum and pseudospectra of $A$ are exactly fourfold symmetric, as one can prove by showing that $A$ is unitarily similar to i $A$ via a Discrete Fourier Transform matrix: i $A=F A F^{*}$ with $f_{j k}=N^{-1 / 2} \omega^{(j-1)(k-1)}, 1 \leq j, k \leq N$, where $\omega=\exp (-2 \pi \mathrm{i} / N)$. This symmetry is a special feature of this example and is not present in general for the matrices considered in this article.
} 


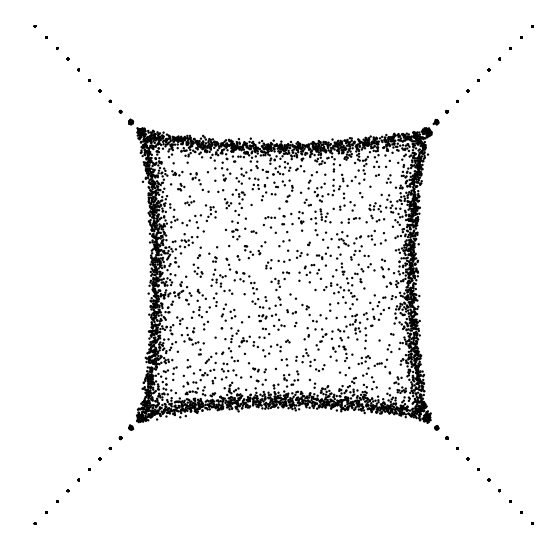

Figure 2: Superposition of the eigenvalues of 100 matrices $A+E$, where each $E$ is a random complex matrix of norm $10^{-4}$.

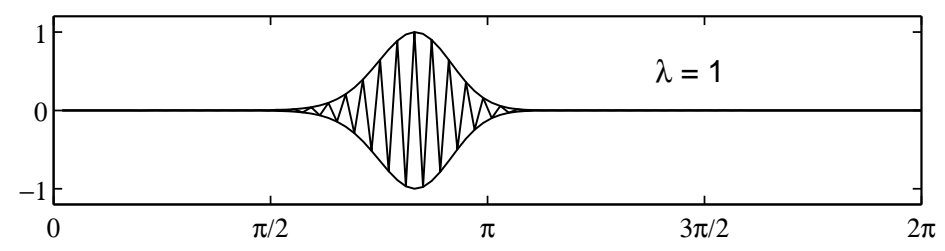

Figure 3: A pseudoeigenvector $v$ of $A$ corresponding to the pseudoeigenvalue $\lambda=1$, with $\|(A-\lambda) v\| /\|v\| \approx 5.688 \times 10^{-6}$. Here and in subsequent such pictures the real part, absolute value, and negative of the absolute value are shown. The data in question are discrete vectors of length $N$, but the dots are connected and thus may appear as smooth curves. The horizontal coordinate is taken as $x_{j}$, ranging from $2 \pi / N$ to $2 \pi$ as $j$ ranges from 1 to $N$.

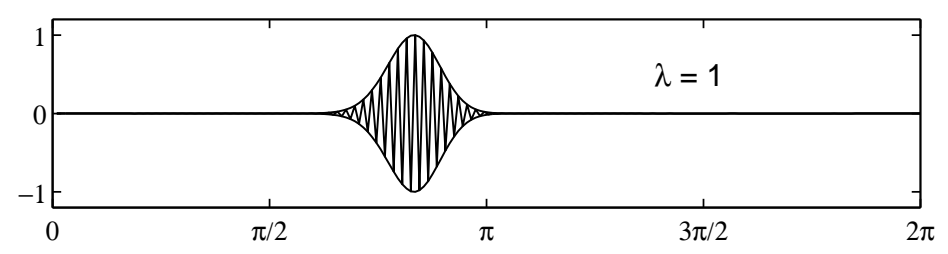

Figure 4: Same as Fig. 3 but with the dimension $N$ increased from 101 to 201. The value of $\varepsilon$ is approximately squared, with $\varepsilon \approx 9.949 \times 10^{-11}$, and the width of the wave packet is reduced by a factor of approximately $\sqrt{2}$.

per wavelength. We shall see that $k_{*}, x_{*}$, and $\lambda$ are related by the condition

$$
f\left(x_{*}, k_{*}\right)=\lambda,
$$

where $f(x, k)=2 \sin x-2 \mathrm{i} \sin k$. Figure 4 shows the corresponding narrower wave packet for a matrix of approximately doubled dimension.

The subject of this paper is the analysis of pseudomodes and pseudospectra of matrices like $A$, nonnormal matrices that combine dependence on $x$ and on $k$. We shall define the symbol $f(x, k)$ of such a matrix and show that 
every $\lambda$ in a subset of $\mathbf{C}$ determined by $f(x, k)$ is an $\varepsilon$-pseudoeigenvalue for a value of $\varepsilon$ that shrinks exponentially as $N \rightarrow \infty$. Associated with these pseudoeigenvalues are pseudoeigenvectors in the form of localized wave packets. In an application, these pseudomodes might prove effectively indistinguishable from the true eigenmodes (which typically have the same wave packet form), and of equal physical significance.

There is a literature of pseudospectra of nonnormal true Toeplitz matrices, whose symbol $f$ is independent of $x[6,28,29,34]$. For a matrix of this kind, the nonnormality is introduced only at the boundaries, since if the matrix were circulant instead of Toeplitz, it would be normal. Nevertheless we again have $\varepsilon$-pseudoeigenvalues for exponentially small values of $\varepsilon$, with the associated pseudoeigenvectors decaying exponentially away from the left or right boundary. In Corollary 1 of Section 7 we obtain the main result of [34] as a special case of the more general theory in this article.

The pseudospectra of nonhermitian Toeplitz matrices with variable coefficients were considered in Section 5 of [34], but without any analysis and without noting that the pseudomodes have the form of wave packets. The classic paper on the spectra of variable coefficient Toeplitz matrices in the hermitian case is by Kac, Murdock and Szegő [27].

A close analogue of our results appears in the literature of microlocal analysis of differential and pseudodifferential operators [38]. In several papers beginning in 1996, E. B. Davies has shown that a Schrödinger operator with a complex potential may have wave packet $\varepsilon$-pseudomodes for rapidly decreasing values of $\varepsilon[11,12,13]$. M. Zworski has pointed out that Davies' example and its generalizations are instances of a general theory of variablecoefficient partial differential and pseudodifferential operators developed by Hörmander and others decades ago, originally motivated by the analysis of Lewy's phenomenon of nonexistence of solutions to certain partial differential equations with smooth coefficients $[17,22,24,30,48,49]$.

The methods of microlocal analysis can also applied in the discrete case that is the subject of this paper. After considering a draft of the present article, Borthwick and Uribe have pointed out that the matrices we are investigating can be regarded as Berezin-Toeplitz operators on the two-dimensional torus, operators which arise in geometric quantization of compact symplectic manifolds, and that the microlocal methods imply the existence of wave packet pseudomodes for these operators [5]. These results and those mentioned in the last paragraph, however, depend upon smoothness of the underlying matrix or operator. Here we take a different approach in which smoothness is not required.

\section{Twisted Toeplitz matrices}

We now define the class of matrices for which we shall first establish the existence of exponentially good wave packet pseudomodes. Our theorems assume that the matrices are banded; we do not know to what extent this hypothesis could be weakened.

A Toeplitz matrix is a matrix that is constant along diagonals: for some 
coefficients $\left\{c_{j}\right\}$,

$$
a_{j, \ell}=c_{j-\ell}, \quad 1 \leq j, \ell \leq N .
$$

A circulant matrix is a Toeplitz matrix that extends periodically around the boundaries:

$$
a_{j, \ell}=c_{(j-\ell)(\bmod N)}, \quad 1 \leq j, \ell \leq N .
$$

For integers $m$ and $n$ with $-n \leq m$, we define an $(m, n)$-banded matrix to be a matrix whose nonzero entries all lie within a band extending $m$ entries below the main diagonal and $n$ entries above:

$$
a_{j, \ell} \neq 0 \quad \text { only if } \quad-n \leq j-\ell \leq m .
$$

An $(m, n)$-periodic matrix is the same, except that the nonzero entries wrap around periodically:

$$
a_{j, \ell} \neq 0 \quad \text { only if } \quad-n \leq j-\ell \leq m(\bmod N) .
$$

The symbol of an $(m, n)$-banded Toeplitz matrix or an $(m, n)$-periodic circulant matrix is the $2 \pi$-periodic trigonometric polynomial

$$
f(k)=c_{-n} e^{-n \mathrm{i} k}+\cdots+c_{m} e^{m \mathrm{i} k} .
$$

These definitions apply to individual matrices and are reasonably standard. We now move to the less familiar territory of families of matrices of dimensions $N \rightarrow \infty$ obtained by sampling fixed functions along the diagonals. Given integers $m$ and $n$ with $-n \leq m$, suppose we have $m+n+1$ real or complex $2 \pi$-periodic coefficient functions

$$
c_{\ell}(x), \quad-n \leq \ell \leq m ;
$$

we make no assumptions about continuity or smoothness of $c_{\ell}(x)$ except as stated explicitly. Here is the class of matrices we shall initially work with. ${ }^{4}$

Definition 1. Let $c_{-n}, \ldots, c_{m}$ be $2 \pi$-periodic coefficient functions. The associated family of twisted Toeplitz matrices is the set of $(m, n)$-periodic matrices $\left\{A^{(N)}\right\}_{N \geq 1}$ with coefficients

$$
a_{j, \ell}=c_{(j-\ell)(\bmod N)}\left(x_{j}\right),
$$

where

$$
x_{j}=2 \pi j / N, \quad 1 \leq j \leq N .
$$

Note that although we use the word "Toeplitz," the matrices are in fact periodic at this stage of the discussion, and so the term "twisted circulant" might seem more accurate. However, since nothing has been assumed about continuity of the functions $\left\{c_{j}(x)\right\}$, the periodicity in our definition is mainly

\footnotetext{
${ }^{4}$ Tilli has defined a more general class of "locally Toeplitz" matrices in [39], building on related work by Tyrtyshnikov [43], in which matrices are investigated that can be represented locally as perturbations of Toeplitz matrices by matrices of small rank plus matrices of small norm. We take a step in this direction in Theorem 2 of Section 7.
} 
just formal. The phenomena to be considered and the conditions of our theorems are localized in $x$, applying regardless of whether or not the matrices are periodic in a more substantive sense, and in Section 7 we shall loosen the definitions to require only the local structure that is the essence of the matter (Definition 4).

Given $N$ and $k \in \mathbf{C}$, consider the vector

$$
v=\left(e^{-\mathrm{i} k}, e^{-2 \mathrm{i} k}, \ldots, e^{-N \mathrm{i} k}\right)^{T} .
$$

The $j$ th entry of the matrix-vector product $A^{(N)} v$ can be written

$$
\left(A^{(N)} v\right)_{j}=\left[c_{-n}\left(x_{j}\right) e^{-n \mathrm{i} k}+\cdots+c_{m}\left(x_{j}\right) e^{m \mathrm{i} k}\right] v_{j} .
$$

In other words, we have

$$
\left(A^{(N)} v\right)_{j}=f\left(x_{j}, k\right) v_{j}
$$

where $f$ is the $x$-dependent symbol defined as follows. (The term "symbol" is standard in the literature of pseudo-difference or pseudo-translation operators $[9,31,36,44]$.)

Definition 2. The symbol of the family of twisted Toeplitz matrices associated with the coefficient functions $c_{-n}, \ldots, c_{m}$ is the function

$$
f(x, k)=c_{-n}(x) e^{-n \mathrm{i} k}+\cdots+c_{m}(x) e^{m \mathrm{i} k},
$$

defined for $x \in \mathbf{R}$ and $k \in \mathbf{C}$.

By definition, $f(x, k)$ is $2 \pi$-periodic in both $x$ and $k$. The assumption of bandedness implies that for each fixed $x, f(x, k)$ depends smoothly on $k$. Indeed, it is a trigonometric polynomial and thus an entire function of $k$, i.e., analytic throughout the complex $k$-plane. As for the dependence of $f$ on $x$, in Theorems 1-3 we shall need nothing more than differentiability at a single point $x_{*}$, and in Theorems $4-5$ we shall require even less. To construct pseudomodes explicitly by WKBJ expansion or related methods, more derivatives are needed (Section 8).

\section{Wave packets and the twist condition}

Suppose that $\left\{A^{(N)}\right\}$ is a family of $(m, n)$-periodic twisted Toeplitz matrices with symbol $f(x, k)$. Let $x_{*}$ and $k_{*}$ be real numbers, and define $\lambda=f\left(x_{*}, k_{*}\right)$. If $f$ were independent of $x$, then $A^{(N)}$ would be a circulant matrix and the vector

$$
v=\left(e^{-\mathrm{i} k_{*}}, e^{-2 \mathrm{i} k_{*}}, \ldots, e^{-N \mathrm{i} k_{*}}\right)^{T}
$$

would be an eigenvector of $A^{(N)}$ with eigenvalue $\lambda$ provided that $N k_{*} / 2 \pi$ is an integer. This is of course not a wave packet but a global vector.

If $f$ varies with $x$, however, (7) is no longer an eigenvector. We could modify it by the method of WKBJ analysis [4], as follows. Let a vector $v$ be defined by

$$
v_{j}=\alpha\left(x_{j}\right) e^{-\mathrm{i} N \phi\left(x_{j}\right)},
$$




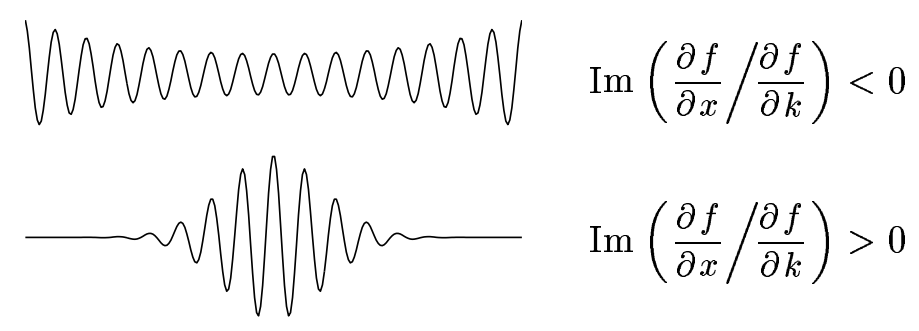

Figure 5: For a wave packet exponentially localized at $x \approx x_{*}, k \approx k_{*}$, the symbol $f(x, k)$ must satisfy the twist condition (10).

where $\alpha$ is continuous and $\phi$ is twice continuously differentiable. Then $v$ is an eigenfunction of $A^{(N)}$ with eigenvalue $\lambda$ if

$$
\left(A^{(N)} v\right)_{j}=\sum_{p=-n}^{m} c_{p}\left(x_{j}\right) \alpha\left(x_{j+p}\right) e^{-\mathrm{i} N \phi\left(x_{j+p}\right)}=\lambda \alpha\left(x_{j}\right) e^{-\mathrm{i} N \phi\left(x_{j}\right)}
$$

for all $j$. Expanding this equation for large $N$ we find that at leading order $\phi$ must satisfy the eikonal equation

$$
\sum_{p=-n}^{m} c_{p}\left(x_{j}\right) e^{-\mathrm{i} p \phi^{\prime}\left(x_{j}\right)}=f\left(x_{j}, \phi^{\prime}\left(x_{j}\right)\right)=\lambda
$$

for all $j$. This result confirms that locally, the wave number $k=\phi^{\prime}$ must satisfy $f(x, k)=\lambda$. Now for (8) to have a localized wave packet at a point $x_{*}, \operatorname{Im}(\phi)$ must have a minimum there, which will happen if $\operatorname{Im}\left(\phi^{\prime}\left(x_{*}\right)\right)=0$ (implying that $k_{*}=\phi^{\prime}\left(x_{*}\right)$ is real) and $\operatorname{Im}\left(\phi^{\prime \prime}\left(x_{*}\right)\right)<0$. Differentiating (9) with respect to $x$ (or expanding the differences about $x_{*}$ ) gives

$$
\frac{\partial f}{\partial x}\left(x_{*}, k_{*}\right)+\frac{\partial f}{\partial k}\left(x_{*}, k_{*}\right) \phi^{\prime \prime}\left(x_{*}\right)=0,
$$

so that

$$
\phi^{\prime \prime}\left(x_{*}\right)=-\frac{\partial f}{\partial x} / \frac{\partial f}{\partial k} .
$$

Thus again we see that if $\operatorname{Im}\left(\frac{\partial f}{\partial x} / \frac{\partial f}{\partial k}\right)>0$, then $v$ has a local maximum at $x_{*}$ and forms a localized wave packet, whereas if $\operatorname{Im}\left(\frac{\partial f}{\partial x} / \frac{\partial f}{\partial k}\right)<0$, then it has a local minimum and grows exponentially away from $x_{*}$.

We formalize these conditions as follows (Fig. 5).

Definition 3. Let $f(x, k)$ be a function of $x \in \mathbf{R}$ and $k \in \mathbf{C}$ that is $2 \pi$ periodic in both variables, and let $x_{*}$ and $k_{*}$ be real numbers. Then $f$ satisfies the twist condition at $x=x_{*}, k=k_{*}$ if at this point it is differentiable with respect to $x$ with $\partial f / \partial k \neq 0$ and

$$
\operatorname{Im}\left(\frac{\partial f}{\partial x} / \frac{\partial f}{\partial k}\right)>0 .
$$

It satisfies the antitwist condition if it has the same properties with (10) replaced by

$$
\operatorname{Im}\left(\frac{\partial f}{\partial x} / \frac{\partial f}{\partial k}\right)<0
$$


Our twist and antitwist conditions are matrix analogues of Hörmander's commutator condition for differential and pseudodifferential operators [22,23,24], which in the general form used in microlocal analysis becomes a condition involving the Poisson bracket of a principal symbol, $\{\operatorname{Re}(f), \operatorname{Im}(f)\}$; see for example Theorem 3.3.7 of [23]. Connections with pseudospectra are pointed out in $[14,48,49]$, and for the discrete case of Berezin-Toeplitz operators mentioned in the Introduction, see [5]. In Section 6 we shall reformulate the twist and antitwist conditions in terms of winding numbers of the symbol $f(x, k)$ acting on $k \in[0,2 \pi]$ for each fixed $x$.

\section{Main theorem}

Our purpose is to show that if the twist condition is satisfied at $x_{*}, k_{*}$, together with some other conditions, then there exist exponentially good wave packet pseudomodes there. No smoothness of the symbol is required beyond the differentiability at a point that is part of the twist condition. Here is our main theorem; it will be generalized considerably in Section 7 .

Theorem 1. Let $\left\{A^{(N)}\right\}$ be a family of $(m, n)$-periodic twisted Toeplitz matrices with symbol $f(x, k)$. Let $x_{*}$ and $k_{*}$ be real numbers, define $\lambda=$ $f\left(x_{*}, k_{*}\right)$, and suppose that the twist condition (10) is satisfied at $x=x_{*}$, $k=k_{*}$. Suppose moreover that $f\left(x_{*}, k\right) \neq \lambda$ for all real $k \not \equiv k_{*}(\bmod 2 \pi)$ and that the extreme coefficients are nonzero in the following sense: if $n>0$, $c_{-n}\left(x_{*}\right) \neq 0$; if $m>0, c_{m}\left(x_{*}\right) \neq 0$; if $m=0$ or $n=0, c_{0}\left(x_{*}\right) \neq \lambda$; if $n<0$ or $m<0, \lambda \neq 0$. Then there exist constants $C_{1}, C_{2}>0$ and $M>1$ such that for all sufficiently large $N$ there exists a nonzero pseudoeigenmode $v^{(N)}$ that is exponentially good,

$$
\frac{\left\|\left(A^{(N)}-\lambda\right) v^{(N)}\right\|}{\left\|v^{(N)}\right\|} \leq M^{-N}
$$

and localized,

$$
\frac{\left|v_{j}^{(N)}\right|}{\max _{j}\left|v_{j}^{(N)}\right|} \leq C_{1} \exp \left(-C_{2} N\left(x_{j}-x_{*}\right)^{2}\right) \quad(\bmod 2 \pi) .
$$

The condition that $f\left(x_{*}, k\right) \neq \lambda$ for all real $k \not \equiv k_{*}(\bmod 2 \pi)$ might easily be overlooked, but it is important: as will become clear in Sections 7 and 8 , it is the price we pay for not requiring $f$ to be smooth. The meaning of " $\bmod 2 \pi "$ in (13) is that the inequality holds with $x_{j}$ adjusted by multiples of $2 \pi$ as necessary so that $x_{j}-x_{*} \in[-\pi, \pi]$.

The proof of Theorem 1 occupies the remainder of this section. The essential idea, involving a nonempty intersection of subspaces of local modes decaying to the left and the right, is summarized in the two paragraphs at the end of this section and reappears throughout Section 7; see in particular Fig. 15 and the proof of Theorem 5 . 
Suppose $v=\left(v_{1}, \ldots, v_{N}\right)^{T}$ is an eigenvector of $A^{(N)}$ with eigenvalue $\lambda$, i.e., $\left(A^{(N)}-\lambda\right) v=0$. Then assuming $m, n \geq 0$, for each $j$ we have

$$
c_{m}\left(x_{j}\right) v_{j-m}+\cdots+c_{-n}\left(x_{j}\right) v_{j+n}=\lambda v_{j} .
$$

(For $m<0$ or $n<0$ the bookkeeping must be modified in straightforward ways that we do not spell out.) This is an $(m+n+1)$-term recurrence equation with variable coefficients. We may associate it with a characteristic polynomial

$$
p(x, z)=c_{m}(x)+c_{m-1}(x) z+\cdots+c_{-n} z^{m+n}-\lambda
$$

with coefficients dependent on $x$. Note that $p\left(x_{j}, z\right)=0$ is the eikonal equation (9) with $e^{i \phi^{\prime}\left(x_{j}\right)}=z$. For any fixed $j, p\left(x_{j}, \cdot\right)$ has $m+n$ roots $\left\{\zeta_{\ell}\right\}$ counted with multiplicity, if we include as many roots $\zeta=\infty$ as there are leading coefficients equal to zero. Each root with $|\zeta|>1$ corresponds to exponential growth of $v_{j}$ as $j$ increases and exponential decay as $j$ decreases; for $|\zeta|<1$ the pattern is reversed. Our choice of $\lambda$ will ensure that one root (and only one) has $|\zeta|=1$ and passes from outside to inside the unit circle as $x$ increases through $x_{*}$, and thus corresponds to exponential decay in both directions. We begin in Lemma 1 , however, with a result based on the assumption that the roots are separated from the unit circle.

Our proof will work with a fixed range of values of $x$, chosen narrow enough so that the coefficients $\left\{c_{\ell}(x)\right\}$ vary sufficiently little so that, by the assumptions on $c_{m}\left(x_{*}\right)$ and $c_{-n}\left(x_{*}\right)$, there are no roots at 0 or $\infty$. With this destination in mind let us first consider the problem of a recurrence relation (14) with constant coefficients and $c_{-n} \neq 0$. We can write such a recurrence in matrix form as

$$
\left(\begin{array}{c}
v_{j+1} \\
v_{j+2} \\
v_{j+3} \\
\vdots \\
v_{j+q}
\end{array}\right)=\left(\begin{array}{ccccc}
0 & 1 & & & \\
& 0 & 1 & & \\
& & 0 & 1 & \\
& & & \ddots & \ddots \\
e_{0} & e_{1} & e_{2} & \ldots & e_{q-1}
\end{array}\right)\left(\begin{array}{c}
v_{j} \\
v_{j+1} \\
v_{j+2} \\
\vdots \\
v_{j+q-1}
\end{array}\right)
$$

where $q=m+n$ and $e_{\ell}=-c_{m-\ell} / c_{-n}$ (with the special case $e_{m}=(\lambda-$ $\left.\left.c_{0}\right) / c_{-n}\right)$, or more compactly, with $v^{(j)}=\left(v_{j}, \ldots, v_{j+q-1}\right)^{T}$,

$$
v^{(j+1)}=C v^{(j)},
$$

where $C$ is the "transfer matrix" of (16). For $\zeta \neq 0$, such an equation has a solution of the form

$$
v_{j}=\zeta^{j} \quad \forall j,
$$

implying

$$
v^{(j)}=\zeta^{j} v^{(0)},
$$

if and only if $\zeta$ satisfies

$$
p(\zeta)=0,
$$


where $p$ is the polynomial $p(x, z)$ of (15) but with the first argument removed since the coefficients are constant.

Now consider (19) but with varying coefficients. That is, let $E_{j}$ be a sequence of $q \times q$ matrices, which we shall eventually require to have sufficiently small entries, and consider

$$
v^{(j)}=\left(C+E_{j}\right) \cdots\left(C+E_{1}\right) v^{(0)} .
$$

We first show that under suitable hypotheses, these products attenuate and amplify certain vectors exponentially. This is a basic result with the flavor of stable and unstable manifolds in dynamical systems; see [25] and Appendix II of [35]. Afterwards we shall refine it to a more specialized result, Lemma 2, that is actually the one needed to prove Theorem 1 . Lemma 1 itself will be applied later in the proof of Theorem 4.

Lemma 1. Let $C$ be a nonsingular $(\eta+\nu) \times(\eta+\nu)$ matrix $(\eta, \nu \geq 0$, $\eta+\nu \geq 1)$ that has $\eta$ eigenvalues with $|\zeta|<\rho<1$ and $\nu$ eigenvalues with $|\zeta|>R>1$, and consider products of the form

$$
S^{(J)}=\left(C+E_{J}\right) \cdots\left(C+E_{1}\right)
$$

with $\left\|E_{j}\right\|<\varepsilon$ for each $j$. There exists $\varepsilon>0$, independent of $J$, such that these products separate $\mathbf{C}^{\eta+\nu}$ into exponentially amplified and attenuated subspaces in the following sense: for each sufficiently large $J$, there is a $\eta$-dimensional subspace $\mathcal{S}_{\eta} \subseteq \mathbf{C}^{\eta+\nu}$ such that

$$
\left\|S^{(J)} u\right\| \leq \rho^{J}\|u\| \quad \forall u \in \mathcal{S}_{\eta}
$$

and a $\nu$-dimensional subspace $\mathcal{S}_{\nu} \subseteq \mathbf{C}^{\eta+\nu}$ such that

$$
\left\|S^{(J)} u\right\| \geq R^{J}\|u\| \quad \forall u \in \mathcal{S}_{\nu} .
$$

Proof. We shall see that $\mathcal{S}_{\nu}$ can be chosen independently of $J$, since almost all vectors lead to exponential growth, but $\mathcal{S}_{\eta}$ must depend on $J$, since exponential decay is more delicate.

The first step is to reduce $C$ to block-diagonal form. There exists a nonsingular matrix $X$ such that

$$
X C X^{-1}=\left(\begin{array}{cc}
D_{\nu} & 0 \\
0 & D_{\eta}
\end{array}\right)
$$

where $D_{\nu}$ has dimension $\nu$ and amplifies all $\nu$-vectors,

$$
\left\|D_{\nu} v\right\| \geq T\|v\| \quad \forall v \in \mathbf{C}^{\nu}
$$

and $D_{\eta}$ has dimension $\eta$ and shrinks all $\eta$-vectors,

$$
\left\|D_{\eta} w\right\| \leq \tau\|w\| \quad \forall w \in \mathbf{C}^{\nu} ;
$$

we may choose any $T>R$ that is smaller than the eigenvalues of $C$ outside the unit circle, and any $\tau<\rho$ that is larger than the eigenvalues of $C$ inside 
the unit circle. If each term $C+E_{j}$ in (21) is transformed to $X\left(C+E_{j}\right) X^{-1}$, then the intermediate pairs $X^{-1} X$ cancel so that only the leftmost $X$ and rightmost $X^{-1}$ remain, which have no effect on the large- $J$ assertion. Since $E_{j}$ becomes $X E_{j} X^{-1}$ in this process, the norm bound $\varepsilon$ has to be adjusted by the condition number $\kappa(X)=\|X\|\left\|X^{-1}\right\|$. All this is straightforward, and rather than encumber the rest of the argument with $X$ and associated details, let us assume from now on, without loss of generality, that $C$ itself has block diagonal form to begin with,

$$
C=\left(\begin{array}{cc}
D_{\nu} & 0 \\
0 & D_{\eta}
\end{array}\right),
$$

with $D_{\nu}$ and $D_{\eta}$ satisfying (24) and (25).

We identify first a growing space $S_{\nu}$. Define $\delta, \varepsilon>0$ by

$$
\delta \leq \frac{T-R}{T}, \quad \varepsilon=\frac{\delta(T-\tau)}{4},
$$

and let $\mathcal{G} \subseteq \mathbf{C}^{\eta+\nu}$ denote the cone of all vectors $u=\left(v^{T}, w^{T}\right)^{T} \in \mathbf{C}^{\eta+\nu}$ with the property

$$
\|w\| \leq \delta\|v\| .
$$

Consider what happens when $C+E_{j}$, with $\left\|E_{j}\right\| \leq \varepsilon$, multiplies a vector $u=\left(v^{T}, w^{T}\right)^{T} \in \mathcal{G}:$

$$
\left(\begin{array}{c}
v^{\prime} \\
w^{\prime}
\end{array}\right)=\left(C+E_{j}\right)\left(\begin{array}{c}
v \\
w
\end{array}\right)
$$

assuming (without loss of generality) $\|v\|=1$. We calculate

$$
\left\|v^{\prime}\right\| \geq T-2 \varepsilon=T-\frac{\delta}{2}(T-\tau)=\left(\frac{1}{2}-\frac{\delta}{2}\right)(T-\tau)+\frac{1}{2}(T+\tau)>\frac{1}{2}(T+\tau)
$$

whereas

$$
\left\|w^{\prime}\right\| \leq \tau \delta+2 \varepsilon=\tau \delta+\frac{\delta}{2}(T-\tau)=\frac{\delta}{2}(T+\tau) .
$$

Thus we have $\left\|w^{\prime}\right\| \leq \delta\left\|v^{\prime}\right\|$, showing that $C+E_{j}$ maps $\mathcal{G}$ into itself. Moreover since $\left\|v^{\prime}\right\|>T-T \delta / 2$ we have

$$
\left\|v^{\prime}\right\|-R>T-R-T \delta+\frac{T \delta}{2} \geq \frac{T \delta}{2}
$$

since $T \delta \leq T-R$, which implies that each time a term $\left(C+E_{j}\right)$ multiplies a vector $u=\left(v^{T}, w^{T}\right)^{T} \in \mathcal{G}$, the norm of the upper part increases by a factor of at least $R(1+T \delta / 2)$. Putting these observations together we find that if $u \in \mathcal{G}$, then $S^{(J)} u \in \mathcal{G}$ for all $J$ and $\left\|S^{(J)} u\right\| \geq R^{J}\|u\|$ for all sufficiently large $J$. Finally, we note that $\mathcal{G}$ contains the $\nu$-dimensional invariant subspace associated with $D_{\nu}$, i.e., the subspace spanned by the $\nu$ vectors $e_{1}, e_{2}, \ldots, e_{\nu} \in \mathbf{C}^{\eta+\nu}$. Thus this subspace is a suitable choice of $S_{\nu}$.

Next we find a decaying space $S_{\eta}$. For this we can invert the series (21) to get

$$
\left(S^{(J)}\right)^{-1}=\left(C+E_{1}\right)^{-1} \cdots\left(C+E_{J}\right)^{-1}=\left(C^{-1}+\tilde{E}_{1}\right) \cdots\left(C^{-1}+\tilde{E}_{J}\right)
$$


with

$$
\tilde{E}_{j}=-\left(C+E_{j}\right)^{-1} E_{j} C^{-1} .
$$

(It is here that we have used the assumption that $C$ is nonsingular, which implies that $C+E_{j}$ is nonsingular too if $\varepsilon$ is sufficiently small.) By the same argument as before, $\left(S^{(J)}\right)^{-1}$ has a growing space $\mathcal{T}$ of dimension $\eta$, and we take $\mathcal{S}_{\eta}=\left(S^{(J)}\right)^{-1} \mathcal{T}$.

Other papers that have considered perturbed powers of matrices include [21] and [37], though the details are quite different from ours.

The next lemma is a more specialized variant of Lemma 1 designed for recurrences that have a root passing through the unit circle, as occurs at the center of a wave packet pseudomode.

Lemma 2. Let $\{C(x)\}$ be a family of nonsingular $(\eta+\nu+1) \times(\eta+\nu+1)$ matrices $(\eta, \nu \geq 0)$ depending differentiably on $x$ at $x=x_{*}$. Assume that $C\left(x_{*}\right)$ has $\eta$ eigenvalues with $|\zeta|<\rho<1$, $\nu$ eigenvalues with $|\zeta|>R>1$, and one eigenvalue $\mu_{*}$ with $\left|\mu_{*}\right|=1$. Assume further that $d|\mu| / d x<0$ at $x=x_{*}$, where $\mu=\mu(x)$ is the eigenvalue of $C(x)$ that converges to $\mu_{*}$ as $x \rightarrow x_{*}$. For any $\Delta x>0$ and $J \geq 1$, consider the product

$$
S^{(J)}=C\left(x_{*}+(J-1) \Delta x / J\right) \cdots C\left(x_{*}+\Delta x / J\right) C\left(x_{*}\right) .
$$

There exist $\Delta x>0$ and $M>1$, independent of $J$, such that these products separate $\mathbf{C}^{\eta+\nu+1}$ into exponentially amplified and attenuated subspaces in the following sense: for each sufficiently large $J$, there is a $(\eta+1)$-dimensional subspace $\mathcal{S}_{\eta} \subseteq \mathbf{C}^{\eta+\nu+1}$ such that

$$
\left\|S^{(J)} u\right\| \leq M^{-J}\|u\| \quad \forall u \in \mathcal{S}_{\eta}
$$

and a $\nu$-dimensional subspace $\mathcal{S}_{\nu} \subseteq \mathbf{C}^{\eta+\nu+1}$ such that

$$
\left\|S^{(J)} u\right\| \geq M^{J}\|u\| \quad \forall u \in \mathcal{S}_{\nu} .
$$

Proof. It is only (27) that we shall need, not (28), so this is the claim that we shall prove. As in the final paragraph of the proof of Lemma 1, to show that $S^{(J)}$ has a decaying subspace of dimension $\eta+1$, we shall show that

$$
\left(S^{(J)}\right)^{-1}=C\left(x_{*}\right)^{-1} \cdots C\left(x_{*}+(J-1) \Delta x / J\right)^{-1}
$$

has a growing subspace of this dimension. Our first step, as in the proof of Lemma 1, is to assume without loss of generality that $C\left(x_{*}\right)^{-1}$ has the block-diagonal form

$$
C\left(x_{*}\right)^{-1}=\left(\begin{array}{ccc}
D_{\eta} & 0 & 0 \\
0 & \mu_{*}^{-1} & 0 \\
0 & 0 & D_{\nu}
\end{array}\right),
$$

where $D_{\eta}$ has dimension $\eta$ and amplifies all $\eta$-vectors,

$$
\left\|D_{\eta} v\right\| \geq T\|v\| \quad \forall v \in \mathbf{C}^{\eta}
$$


$D_{\nu}$ has dimension $\nu$ and shrinks all $\nu$-vectors,

$$
\left\|D_{\nu} w\right\| \leq \tau\|w\| \quad \forall w \in \mathbf{C}^{\nu},
$$

and $\left|\mu_{*}^{-1}\right|=1$. (Here $\tau<1<T$.)

Without loss of generality we simplify the discussion by assuming $x_{*}=0$.

The proof rests on an observation of linear algebra: if a diagonal matrix is perturbed by $O(\varepsilon)$ in the off-diagonal positions, its eigenvalues of multiplicity 1 change by only $O\left(\varepsilon^{2}\right)$. (One can prove this by considering the characteristic polynomial.) We can exploit this phenomenon as follows. Our task is to consider the product of a set of matrices

$$
C(x)^{-1}=\left(\begin{array}{ccc}
D_{\eta} & 0 & 0 \\
0 & c(x) & 0 \\
0 & 0 & D_{\nu}
\end{array}\right)+E(x)
$$

with $\|E(x)\|=O(x)$, where $E(x)$ is constructed to have a zero entry in the middle position $(\eta+1, \eta+1)$ and $c(x)$ is defined accordingly. Since $\mu(x)^{-1}=\mu_{*}^{-1}+x\left(d \mu^{-1} / d x\right)\left(x_{*}\right)+o(x)$, it follows from the fact of linear algebra just mentioned that $c(x)=\mu_{*}^{-1}+x\left(d \mu^{-1} / d x\right)\left(x_{*}\right)+o(x)$ too. Choose $\gamma$ to be a constant in the range $0<\gamma<d\left|\mu^{-1}\right| / d x$, and collect the upper-left $(\eta+1) \times(\eta+1)$ block of $C(x)^{-1}$ into a single matrix $D_{\eta+1}(x)$. Then for some $\sigma>0$ and $x_{\max }>0$ we have

$$
C(x)^{-1}=\left(\begin{array}{cc}
D_{\eta+1}(x) & E_{1}(x) \\
E_{2}(x) & D_{\nu}(x)
\end{array}\right), \quad\left\|E_{1}\right\|,\left\|E_{2}\right\| \leq \sigma x
$$

and

$$
\begin{gathered}
\left\|D_{\eta+1}(x) v\right\| \geq(1+\gamma x)\|v\| \quad \forall v \in \mathbf{C}^{\eta+1}, \\
\left\|D_{\nu}(x) w\right\| \leq \tilde{\tau}\|w\| \quad \forall w \in \mathbf{C}^{\nu}
\end{gathered}
$$

for some $\tilde{\tau}$ with $0<\tilde{\tau}<\tau$ and all $x$ with $0 \leq x \leq x_{\max }$. We now follow estimates as in the proof of Lemma 1. Define

$$
\delta \leq \frac{\gamma}{2 \sigma}, \quad \Delta x=\min \left\{x_{\max }, \frac{\delta(1-\tilde{\tau})}{\sigma}\right\} .
$$

Let $\mathcal{G} \subseteq \mathbf{C}^{\eta+\nu+1}$ be the cone of all vectors $u=\left(v^{T}, w^{T}\right)^{T} \in \mathbf{C}^{\eta+\nu+1}$ with the property

$$
\|w\| \leq \delta\|v\| .
$$

Consider what happens when $C(x)^{-1}$ multiplies a vector $u=\left(v^{T}, w^{T}\right)^{T} \in \mathcal{G}$ :

$$
\left(\begin{array}{c}
v^{\prime} \\
w^{\prime}
\end{array}\right)=\left(\begin{array}{cc}
D_{\eta+1}(x) & E_{1}(x) \\
E_{2}(x) & D_{\nu}(x)
\end{array}\right)\left(\begin{array}{c}
v \\
w
\end{array}\right),
$$

assuming without loss of generality $\|v\|=1$. We calculate

$$
\left\|v^{\prime}\right\| \geq 1+\gamma x-\sigma \delta x \geq 1+\frac{\gamma x}{2}
$$


and

$$
\left\|w^{\prime}\right\| \leq \sigma \Delta x+\tilde{\tau} \delta \leq \delta(1-\tilde{\tau})+\tilde{\tau} \delta=\delta .
$$

These estimates show that $C(x)^{-1}$ maps $\mathcal{G}$ into itself and increases the upper part of the vector by $\left\|v^{\prime}\right\| /\|v\| \geq 1+\gamma x / 2$. Putting a product of such matrices together, we find that if $u \in \mathcal{G}$, then $\left(S^{(J)}\right)^{-1} u \in \mathcal{G}$ for all $J$ and $\left\|\left(S^{(J)}\right)^{-1} u\right\| \geq M^{J}\|u\|$ for all sufficiently large $J$, for some constant $M \approx \exp (\gamma \Delta x / 4)$. Finally, $\mathcal{G}$ contains the $(\eta+1)$-dimensional invariant subspace associated with $D_{\eta+1}$, which is accordingly a suitable choice of $S_{\eta}$.

Proof of Theorem 1. To establish Theorem 1, we construct a wave packet in an interval $\left[x_{*}-\Delta x, x_{*}+\Delta x\right]$ that satisfies the eigenvalue condition (14) exactly in that interval and also decays exponentially in the sense of (27) there. We do this by applying Lemma 2 in both directions, moving both right and left from one grid point to the next starting at the grid point nearest $x=x_{*}$, taking advantage of the equivalence between the variable coefficient recurrence relation (14) and a variable coefficient analogue of the transfer matrix equation (16).

First consider the argument moving from $x_{*}$ to the right. The hypothesis of Theorem 1 that $f\left(x_{*}, k\right) \neq \lambda$ for all real $k \not \equiv k_{*}(\bmod 2 \pi)$ ensures that the transfer matrix $C\left(x_{*}\right)$ has just a single eigenvalue $\mu_{*}$ on the unit circle, as required in Lemma 2. The twist condition of Theorem 1 ensures that $d|\mu| / d x<0$ at $x=x_{*}$, and also also required by that lemma, the hypotheses on the extreme coefficients ensure that $C(x)$ is nonsingular for all $x$ sufficiently close to $x_{*}$. The matrices $S^{(J)}$ are then the solution operators that transfer a set of initial vectors $\left(v_{j}, \ldots v_{j+q-1}\right)$ for grid points near $x_{*} J$ steps to the right. According to Lemma 2, there is an $(\eta+1)$-dimensional subspace $\mathcal{S}_{\eta}^{\text {(right) }} \subseteq \mathbf{C}^{\eta+\nu+1}$ of such initial vectors that generate solutions in $\left[x_{*}, x_{*}+\Delta x\right]$ that decay exponentially in the sense of (27) in that interval. The value of $J$ will grow proportionally to $N(J \approx N \Delta x / 2 \pi)$, and thus the $M^{-J}$ of (27) is equivalent to the $M^{-N}$ needed in (12).

Similarly, by an obvious symmetry, we can apply Lemma 2 moving from $x_{*}$ to the left. By the same arguments, we conclude that there is a $(\nu+1)$ dimensional subspace $\mathcal{S}_{\eta}^{\text {(left) }} \subseteq \mathbf{C}^{\eta+\nu+1}$ of initial vectors vectors that generate solutions in $\left[x_{*}-\Delta x, x_{*}\right]$ that decay exponentially in the analogous sense in $\left[x_{*}-\Delta x, x_{*}\right]$.

Now we take the intersection of these two subspaces. Their dimensions are $\eta+1$ and $\nu+1$, and they are subspaces of $\mathbf{C}^{\eta+\nu+1}$. It follows that $\mathcal{S}_{\eta}^{\text {(right) }} \cap \mathcal{S}_{\eta}^{\text {(left) }}$ is a subspace of dimension at least 1 (in fact, it will be exactly 1) of vectors that generate solutions of the eigenvalue equation in $\left[x_{*}-\Delta x, x_{*}+\Delta x\right]$ that decay exponentially in the sense of (27).

Thus there exists a vector that satisfes the eigenvalue equation in $\left[x_{*}-\right.$ $\left.\Delta x, x_{*}+\Delta x\right]$ and has the necessary exponential decay there. Near $x_{*}-\Delta x$ and $x_{*}+\Delta x$, such a vector will be exponentially small. By extending it by values 0 outside this interval, we obtain a pseudoeigenvector $v^{(N)}$ that satisfies both conditions (12) and (13). 


\section{$5 \quad$ Examples}

In this section we illustrate Theorem 1 with four examples computed numerically with EigTool $[46,47]$. We abbreviate the twist ratio by

$$
\mathcal{T}(x, k)=\frac{\partial f}{\partial x} / \frac{\partial f}{\partial k}(x, k)
$$

and note that the twist condition $(10)$ is that $\mathcal{T}(x, k)$ is well defined at $x_{*}, k_{*}$ and has positive imaginary part.

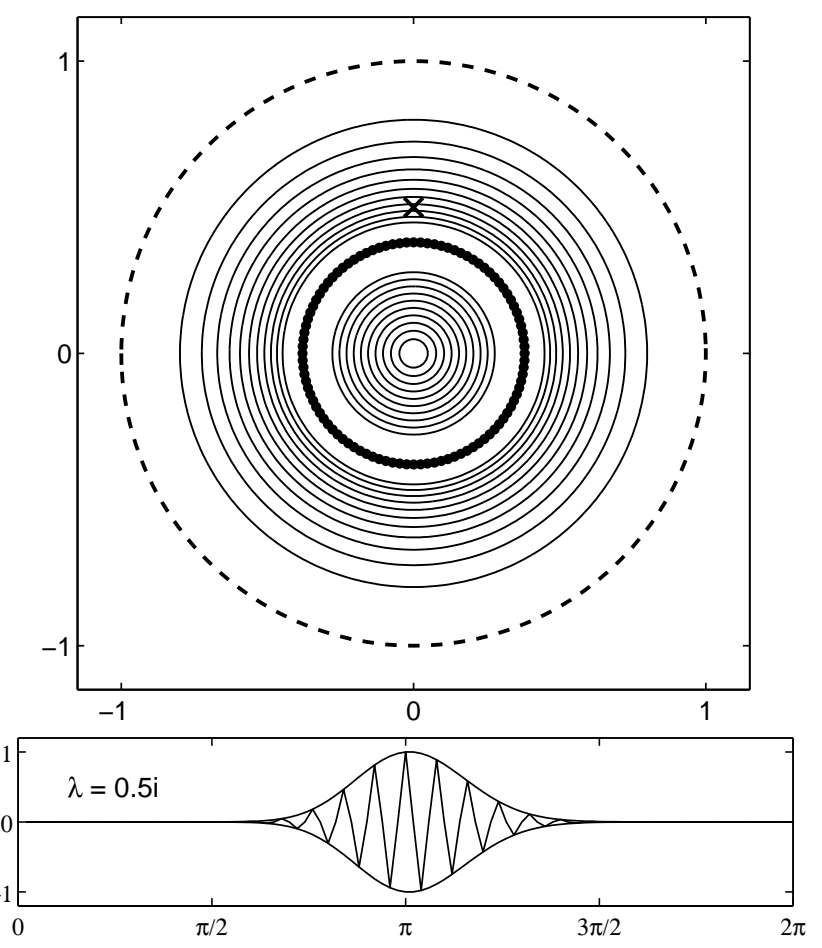

Figure 6: $\varepsilon$-pseudospectra of the "Mt. Fuji matrix" (36) with $N=100, \varepsilon=$ $10^{-2}, 10^{-3}, \ldots, 10^{-12}$ (counting from outside in towards the circle of eigenvalues, or from 0 out towards the circle of eigenvalues). Here and in the following figures, the dashed curve bounds the region in which $\left\|\left(\lambda-A^{(N)}\right)^{-1}\right\|$ grows exponentially as $N \rightarrow \infty$. The lower curve shows the optimal $\varepsilon$-pseudoeigenvector for $\lambda=\mathrm{i} / 2$ (marked by the cross), with $x_{*}=\pi, k_{*}=-\pi / 2$, and $\varepsilon \approx 3.53 \times 10^{-10}$.

First, let $A^{(N)}$ be the $N \times N$ "Mt. Fuji matrix" that is zero everywhere except that the first superdiagonal contains the entries $1 / N, 2 / N, \ldots,(N-$ $1) / N$, continued around periodically to the entry $a_{N 1}=1$. The symbol is

$$
f(x, k)=\frac{x e^{-\mathrm{i} k}}{2 \pi}
$$

with $m=-1$ and $n=1$, and as $x$ and $k$ range over [0,2 $2 \pi, f$ ranges over the unit disk. The twist ratio is

$$
\mathcal{T}(x, k)=\mathrm{i} / x,
$$




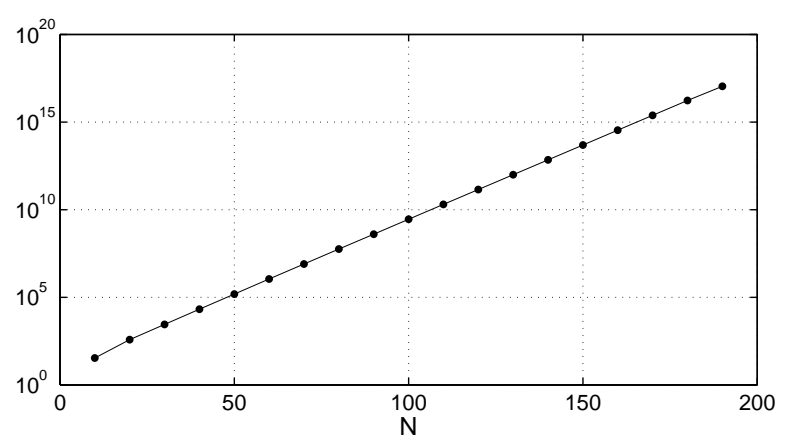

Figure 7: Resolvent norm $\left\|\left(\lambda-A^{(N)}\right)^{-1}\right\|$ against $N$ for the same Mt. Fuji matrices and value $\lambda=\mathrm{i} / 2$ as in Fig. 6 . The exponential growth confirms condition (12) of Theorem 1.

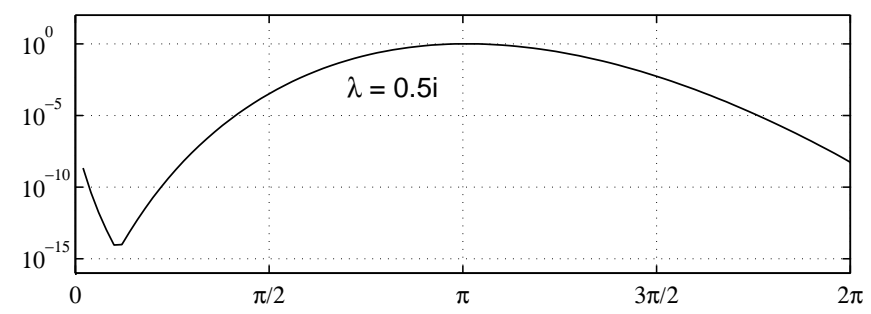

Figure 8: The absolute value of the pseudoeigenvector of Fig. 6, plotted again on a logarithmic scale.

and since $\operatorname{Im} \mathcal{T}(x, k)$ is positive for all $x>0$, we conclude from Theorem 1 that every point $\lambda$ in the punctured unit disk is an exponentially good pseudoeigenvalue. Figure 6 confirms this prediction.

The lower part of Fig. 6 shows a pseudoeigenvector of $A^{(N)}$ corresponding to a particular choice of $\lambda$, namely $\lambda=\mathrm{i} / 2$. Here and in our subsequent such figures (unlike in Figs. 3 and 4), the pseudoeigenvector is an optimal one, a vector $v$ that minimizes $\|(A-\lambda) v\| /\|v\|$. (This is readily computed by means of the singular value decomposition and is the vector produced by EigTool when a pseudomode is requested.) In the figure, we see that the pseudoeigenvector has the form of a wave packet; as always, its real part, absolute value, and negative of absolute value are shown. To relate the packet quantitatively to Theorem 1 we note that from $(36), f(x, k)=\lambda=$ $\mathrm{i} / 2$ will be achieved with $x=\pi$ and $k=-\pi / 2$, and only for these values. This is why the wave packet in Fig. 6 lies at the center of the interval with 4 points per wavelength.

Figure 7 confirms that, as predicted by (12), the resolvent norm at this point in the punctured disk grows exponentially as $N \rightarrow \infty$.

Theorem 1 does not guarantee that an optimal pseudoeigenvector has the form of a wave packet, merely that there exists an exponentially good pseudoeigenvector in that form. Figure 6 suggests, however, that in this case the optimal pseudoeigenvector does have the shape of a wave packet. We can see its shape more fully by looking at this vector on a logarithmic scale. The downward pointing curve of Fig. 8, locally a parabola, is just 

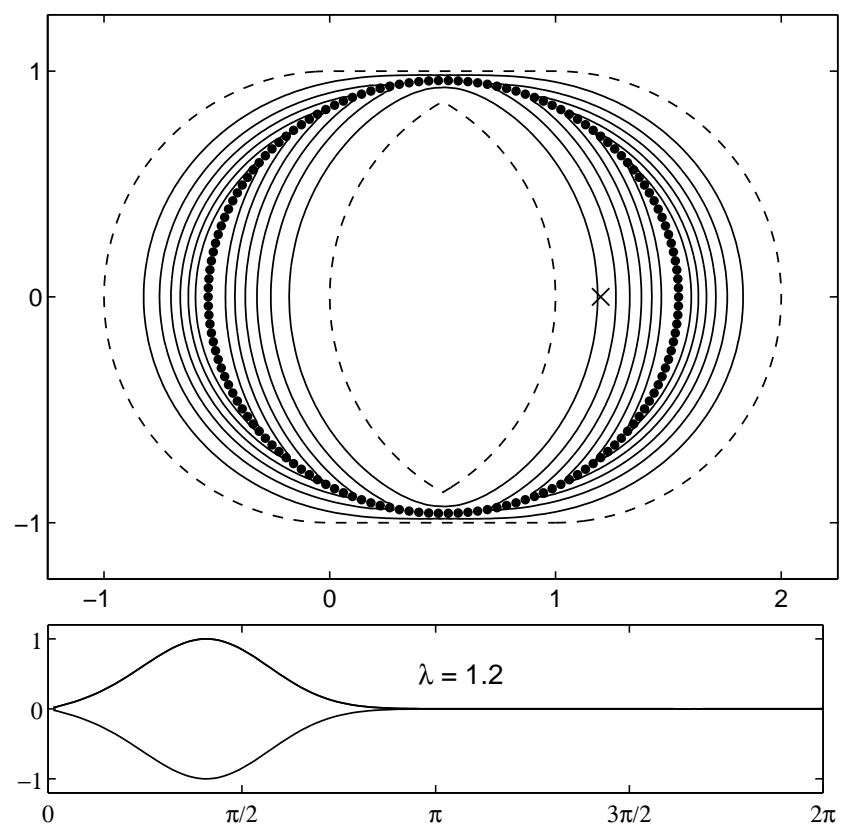

Figure 9: "Wilkinson matrix" (see text) with $N=150, \varepsilon=10^{-2}, 10^{-3}, \ldots, 10^{-7}$. The $\varepsilon$-pseudoeigenvector for $\lambda=1.2$ (cross) has $x_{*}=0.2, k_{*}=0$, and $\varepsilon \approx 0.0073$.

the kind of structure described by (13). We see that the curve reaches a smallest value at $x \approx 0.3$ and then climbs abruptly up again.

Our second example, shown in Figure 9, is a $150 \times 150$ "Wilkinson matrix", consisting of $1 / N, \ldots,(N-1) / N$ on the main diagonal and 1 on the first superdiagonal and also in the $(N, 1)$ position. (Wilkinson proposed a multiple of the nonperiodic version of this matrix with $N=20$ as an example of a matrix with ill-conditioned eigenvalues [45], and pseudospectra were considered in [40].) The symbol is $f(x, k)=x / 2 \pi+e^{-\mathrm{i} k}$, with $m=0$ and $n=1$, and the twist ratio is $\mathcal{T}(x, k)=-\mathrm{i} e^{\mathrm{i} k} / 2 \pi$. This has positive imaginary part for $-\pi / 2<k<\pi / 2$, i.e., whenever $e^{-\mathrm{i} k}$ lies on the right half of the unit circle, $C$. By Theorem 1 , each point $\lambda$ in the crescent-shaped region bounded by $C, C+1$, and the lines $\operatorname{Im} \lambda= \pm 1$ is accordingly an exponentially good pseudoeigenvalue. For the selected value $\lambda=1.2$ we calculate $k_{*}=0$ and $x_{*}=0.4 \pi \approx 1.26$, and this explains why the lower part of Fig. 9 has a wave packet in the left of the interval with no oscillations inside the envelope - the wave packet is purely real.

It is clear from Fig. 9 that the pseudospectra crescent in the half-plane $\operatorname{Re} \lambda \geq \frac{1}{2}$ reflects to an identical pseudospectra crescent in the half-plane $\operatorname{Re} \lambda \leq \frac{1}{2}$. Theorem 1 does not explain this, because the twist condition is not satisfied in this region, but as we shall see in Section 7 (Theorem 3; see also Fig. 16), it is enough for the antitwist condition to be satisfied instead.

Our third example, shown in Figure 10, is a $150 \times 150$ "target matrix", consisting of $-1+x_{j} / \pi$ on the first subdiagonal and 1 on the first superdiagonal, with these patterns continued periodically to $a_{N 1}=1$ and $a_{1 N}=x_{1}$. 

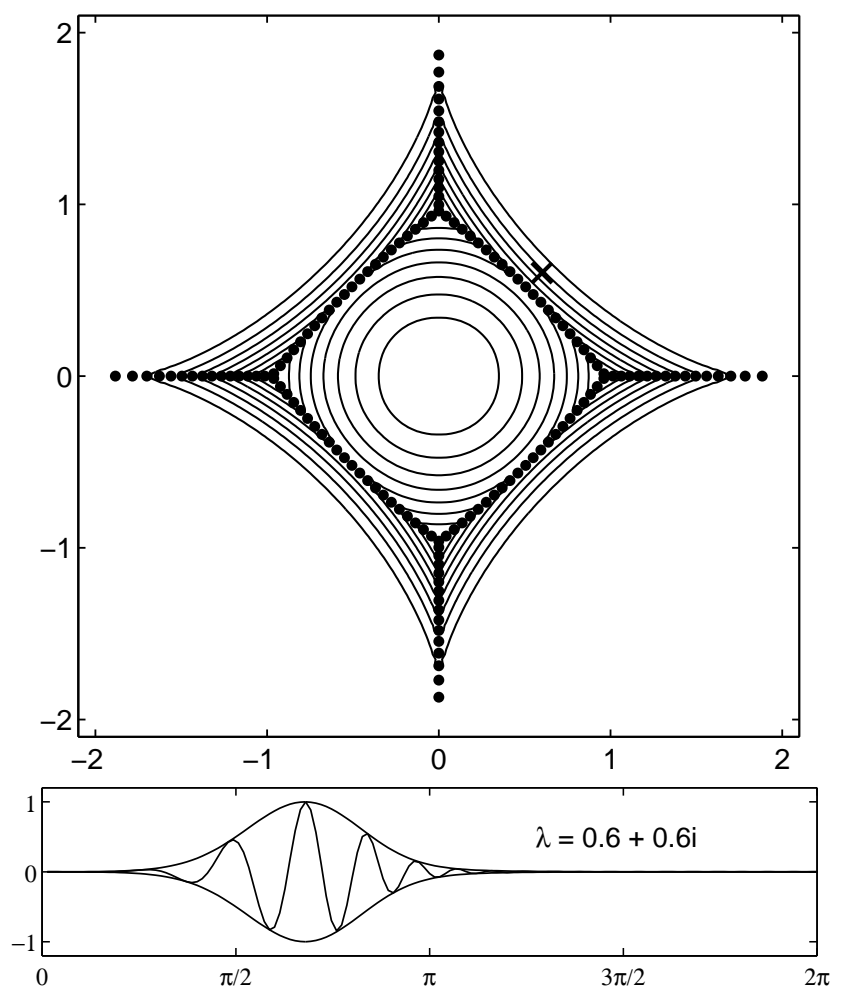

Figure 10: "Target matrix" (38) with $N=150, \varepsilon=10^{-2}, 10^{-3}, \ldots, 10^{-8}$. The $\varepsilon$-pseudoeigenvector for $\lambda=0.6+0.6 \mathrm{i}$ (cross) has $x_{*} \approx 2.11, k_{*} \approx-0.47$, and $\varepsilon \approx 5.30 \times 10^{-4}$.

The symbol is

$$
f(x, k)=e^{-\mathrm{i} k}+\left(-1+\frac{x}{\pi}\right) e^{\mathrm{i} k}=\frac{x}{\pi} \cos k+\mathrm{i}\left(\frac{x}{\pi}-2\right) \sin k .
$$

As $x$ ranges over $[0,2 \pi]$, this function traces all ellipses centered at 0 with axes aligned with the real and imaginary axes and of lengths summing to 4 . For $\lambda=0.6+0.6 \mathrm{i}$, the value of $x$ that satisfies the twist condition is the one corresponding to an ellipse taller than it is wide. A little calculation shows that $x_{*} / \pi$ is the root $\approx 0.67$ of $25 x^{4}-100 x^{3}+82 x^{2}+36 x-36$, leading to $x_{*} \approx 2.11317$ and $k_{*} \approx-0.46904$. This explains why the wave packet in Fig. 10 is located where it is with about $|2 \pi / k| \approx 13.4$ points per wavelength.

Finally, our fourth example illustrates the feature of Theorem 1 that no smoothness of the coefficients is required. We take the same "Mt. Fuji matrix" as in Figs. $6-8$ but add normally distributed random noise of mean zero and standard deviation 0.1 to both the main diagonal and the first superdiagonal (and also the $(N, 1)$ entry). The pseudospectra of the perturbed matrix (not shown) look much like those of the original, and Figure 11 shows that the optimal pseudomode has roughly the same overall form as before too, though it is now quite irregular locally. This random perturbation does not fit the assumptions of Theorem 1: it does not correspond to a welldefined twisted Toeplitz family $\left\{A^{(N)}\right\}$, let alone one with a differentiable symbol at $x=x_{*}$. The fact that there is a wave packet pseudomode any- 

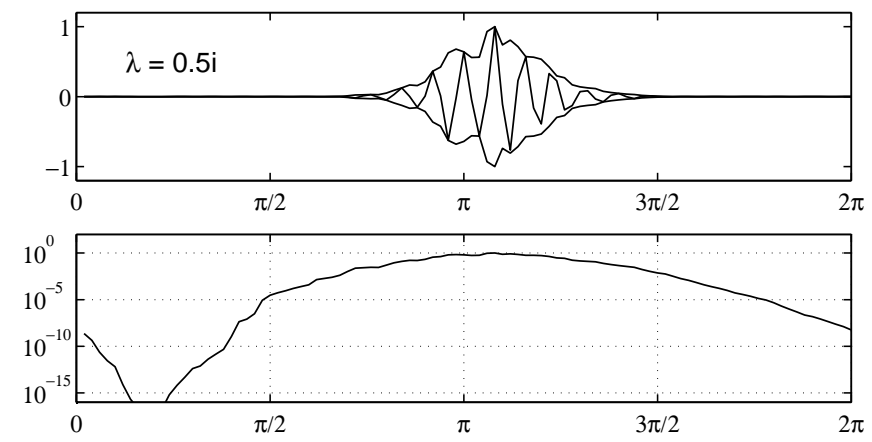

Figure 11: Optimal $\varepsilon$-pseudomode with $\lambda=\mathrm{i} / 2$ (linear and $\log$ scales) for the "Mt. Fuji matrix" of Figs. 6-8, but now with random noise added to the main diagonal and the first superdiagonal. The value of $\varepsilon$ is $4.22 \times 10^{-10}$.

way illustrates how robust this effect is. We shall consider this matter of robustness further in Sections 7 and 8.

\section{Winding number interpretation}

In the theory of Toeplitz matrices and operators, a key role is played by the winding number of the symbol with respect to a point $\lambda \in \mathbf{C}[8,19,34]$. We define this quantity, $I(f, \lambda)$, to be the number of times that the curve $f([0,2 \pi])$ winds around $\lambda$ in the positive sense, where $f$ is the symbol; $I(f, \lambda)$ is undefined if $f([0,2 \pi])$ passes through $\lambda$. Theorem 1 can be interpreted in these terms (Fig. 12). For a twisted Toeplitz matrix, we define $I(f, \lambda, x)$ for each $x$ to be the winding number corresponding to coefficients frozen at that value of $x$. Now suppose $\lambda \in \mathbf{C}$ satisfies $\lambda=f\left(x_{*}, k_{*}\right)$ for some $x_{*}, k_{*} \in[0,2 \pi]$. Then the curve $f\left(x_{*},[0,2 \pi]\right)$ passes through $\lambda$, and thus $I(f, \lambda, x)$ is not defined at $x=x_{*}$. Typically, however, it will be defined for all values of $x$ sufficiently close to $x_{*}$ to the left and right. The twist condition together with the $k \not \equiv k_{*}(\bmod 2 \pi)$ condition of Theorem 1 amounts to the statement that the curve crosses through $\lambda$ in such a way that as $x$ increases through $x_{*}, I(f, \lambda, x)$ decreases by 1 . If $\operatorname{Im}\left(\frac{\partial f}{\partial x} / \frac{\partial f}{\partial k}\right)>0$, then the curve moves in the direction that decreases the winding number. For the antitwist condition, similarly, it moves in the direction that increases the winding number.

Thus Theorem 1 asserts that if the winding number about $\lambda$ decreases as $x$ increases through $x_{*}, \lambda$ is an exponentially good pseudoeigenvalue. Winding numbers have appeared previously in work related to ours - see for example Lemma $3.2^{\prime}$ of [14].

In fact, we can make an explicit connection between the winding number $I=I(f, \lambda, x)$ at a point $x$ where it is defined and the eigenvalues of the associated transfer matrix $C(x)$ of (16). By (6), the rational function $z \mapsto$ $f(x, k)$ with $z=e^{-\mathrm{i} k}$ is a Laurent polynomial with a pole of order $m$ at $z=0$. (We assume $m \geq 0$ for simplicity.) By the principle of the argument of complex analysis, this function has exactly $m-I$ zeros in the unit disk. 


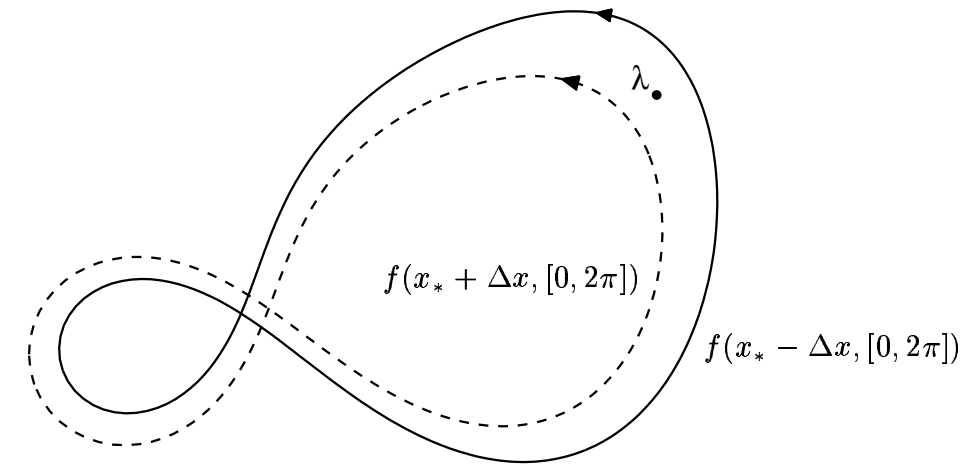

Figure 12: Winding number interpretation of Theorem 1. If the curve $f(x,[0,2 \pi])$ crosses $\lambda$ as $x$ increases through $x_{*}$ in such a way that the winding number about $\lambda$ is decreased, then there is an exponentially good wave packet pseudomode centered at $x_{*}$ with pseudoeigenvalue $\lambda$.

It follows that in Lemma 1, the attenuated and amplified spaces will have dimensions

$$
\eta=m-I, \quad \nu=n+I .
$$

Since our results for twisted Toeplitz matrices depend on winding numbers and so do those of [34] for true Topelitz matrices, one may wonder if there is a connection between the two. Indeed there is: the boundary conditions that make a matrix Toeplitz rather than circulant can be viewed as a special discontinuous case of a "twist". Theorem 4 of the next section makes use of winding numbers to generalize Theorem 1 to discontinuous symbols, and Corollary 1 exhibits Theorem 3.2 of [34] as a consequence of our Theorem 5 .

\section{Generalizations}

Theorem 1 captures the essence of the matter of wave packet pseudomodes, perhaps, but when one comes to concrete examples, it is surprising how often this theorem fails to apply in cases where it "ought" to. Fortunately, it can be extended in many ways.

The most basic generalization, in analogy to the definitions of Tilli [39], is to consider matrices that are not exactly of twisted Toeplitz form as in Definition 1 but close to that form, at least locally near a point $x_{*}$. The following theorem is a second indication (Fig. 11 was the first) that the exponentially strong effects identified in Theorem 1 are structurally stable: they persist under small perturbations. This underlines the fact that Theorem 1 does not depend on smoothness of the symbol.

In the following definition, "near $x_{*}$ " means throughout some real neighborhood of the form $x_{*}-\Delta x<x<x_{*}+\Delta x(\bmod 2 \pi)$, where $\Delta x$ is independent of $N$.

Definition 4. Let $\left\{A^{(N)}\right\}$ be a family of matrices of degrees $N \rightarrow \infty$, let $x_{*} \in[0,2 \pi]$ be fixed, and let $m$ and $n$ be integers with $-n \leq m$. We say 


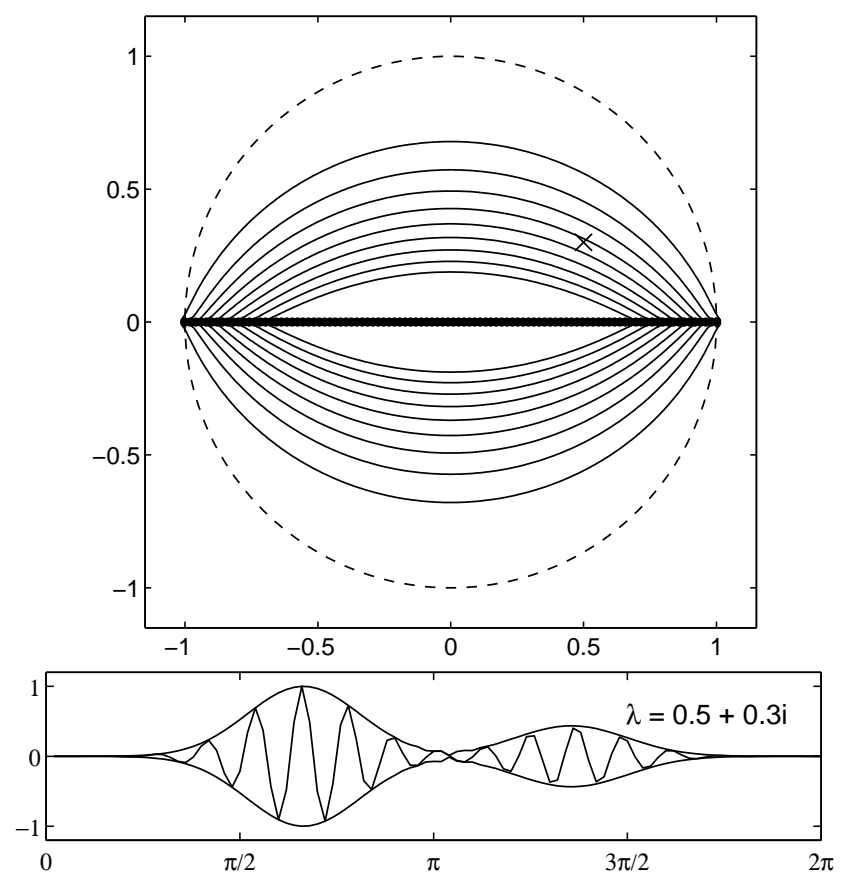

Figure 13: "Ehrenfest matrix" (41) with $N=100, \varepsilon=10^{-2}, 10^{-3}, \ldots, 10^{-10}$. The $\varepsilon$-pseudoeigenvector for $\lambda=0.5+0.3 \mathrm{i}$ (cross) has $\varepsilon \approx 5.71 \times 10^{-6}$. We get a double wave packet because as $x$ increases from 0 to $2 \pi$, the symbol crosses $\lambda$ at two different values of $x$.

that $\left\{A^{(N)}\right\}$ is $(\boldsymbol{m}, \boldsymbol{n})$-periodic near $\boldsymbol{x}_{*}$ if for all sufficiently large $N$, the rows of $A^{(N)}$ corresponding to row indices $j$ with $x_{j}$ near $x_{*}$ are zero outside the $(m, n)$-band. We say that $\left\{A^{(N)}\right\}$ is asymptotically $(\boldsymbol{m}, \boldsymbol{n})$-twisted Toeplitz near $\boldsymbol{x}_{*}$ with symbol $f(x, k)=c_{-n}(x) e^{-n \mathrm{i} k}+\cdots+c_{m}(x) e^{m \mathrm{i} k}$ for some fixed functions $c_{-n}(x), \ldots, c_{m}(x)$ defined near $x_{*}$ if it is $(m, n)$-periodic near $x_{*}$ and for all $j$ with $x_{j}$ near $x_{*}$ and all $\ell$ the coefficients of $A^{(N)}$ satisfy

$$
a_{j, \ell}=c_{(j-\ell)(\bmod N)}\left(x_{j}\right)+o(1)
$$

uniformly as $N \rightarrow \infty$.

Theorem 2. Let $\left\{A^{(N)}\right\}$ be a family of matrices that are asymptotically $(m, n)$-twisted Toeplitz near $x_{*} \in[0,2 \pi]$ with symbol $f(x, k)$ satisfying $\lambda=f\left(x_{*}, k_{*}\right)$ and the other conditions of Theorem 1 at $x=x_{*}$. Then the conclusions (12) and (13) of Theorem 1 hold.

Proof. At the heart of our proof of Theorem 1 are the quantitative estimates of Lemma 2 involving products of matrices $C(x)$ for $x \approx x_{*}$, and in particular, the bounds (32)-(34) on the norms of $E_{1}, E_{2}, D_{\nu+1}$ and $D_{\eta}$. These bounds still hold for matrices $\left\{A^{(N)}\right\}$ satisfying (40), and the proof remains valid in this more general case.

An important special case of Theorem 2 is the situation where the various diagonals of a matrix have different "offsets" from the one implicit in the strict definition (4) of twisted Toeplitz matrices. For example, one may index the matrix entries by columns instead of rows, or diagonally. An 
example of a family of matrices of this kind is shown in Figure $13 .^{5}$ The "Ehrenfest matrix" has $1 /(N-1), 2 /(N-1), \ldots, 1$ on the first superdiagonal and $1,(N-2) /(N-1), \ldots, 1 /(N-1)$ on the first subdiagonal, with symbol

$$
f(x, k)=e^{-\mathrm{i} k}(x / 2 \pi)+e^{\mathrm{i} k}(1-(x / 2 \pi)) .
$$

As in Fig. 10, the set of values covered by $f(x, k)$ is a superposition of ellipses. As $x$ increases from 0 to $2 \pi$, we start with the unit circle with winding number 1 , which then flattens to ellipses with winding number 1 . At $x=\pi$ the ellipse reduces to the interval $[-1,1]$, whereupon it begins to fatten again to ellipses now with winding number -1 until reaching the unit circle once more at $x=2 \pi$. The result is that each point $\lambda$ in the unit disk corresponds to two values $x_{*}$ at which the twist condition is satisfied-first when the winding number jumps from 1 to 0 , then when it jumps again from 0 to -1 . This accounts for the appearance of two wave packets in the optimal pseudomode in the figure.

Theorem 2 also makes it possible for us to extend our results to the antitwist condition.

Theorem 3. Let $\left\{A^{(N)}\right\}$ be a family of matrices as in Theorem 1 or Theorem 2 but such that $f(x, k)$ satisfies the antitwist condition at $x_{*}, k_{*}$ instead of the twist condition. Then $\lambda=f\left(x_{*}, k_{*}\right)$ is again an exponentially good pseudoeigenvalue; the estimates (12) and (13) hold with $A^{(N)}$ replaced by $A^{(N)^{T}}$.

Proof. This is immediate provided we note the fine point that if $\left\{A^{(N)}\right\}$ is a twisted Toeplitz family, then $\left\{A^{(N)^{T}}\right\}$ in general is not, because its diagonals are indexed by columns instead of rows. However, it is asymptotically twisted Toeplitz near any point $x_{*}$ of interest, so Theorem 2 gives the desired conclusion.

Theorem 3 explains the left half of Figure 9, where it is the antitwist rather than the twist condition that is satisfied. Of course, for such a simple problem, one could also devise ad hoc explanations based on the symmetries of the matrix.

Up to here, we have obtained wave packets of type $\exp \left(-N\left(x-x_{*}\right)^{2}\right)$ from eigenvalues of a variable coefficient companion matrix that cross into the unit disk at a point $x=x_{*}$ where the symbol is continuous and differentiable. However, the behavior at $x=x_{*}$ need not be continuous for wave packet pseudomodes to appear. One also gets exponentially good pseudomodes, now of the sharper type $\exp \left(-N\left|x-x_{*}\right|\right)$, if the companion matrix is discontinuous at $x_{*}$ but well-behaved on both sides, with fewer eigenvalues inside the unit disk on the left side than on the right, or equivalently, a larger winding number of the symbol.

Theorem 4. Let $\left\{A^{(N)}\right\}$ be a family of matrices as in Theorems 13 whose symbol $f(x, k)$ is discontinuous at $x_{*}$ but has left- and right-limits $f\left(x_{*}^{-}, k\right)$ and $f\left(x_{*}^{+}, k\right)$ with band widths $\left(m^{-}, n^{-}\right)$and $\left(m^{+}, n^{+}\right)$, and suppose

\footnotetext{
${ }^{5}$ The nonnormal behavior of this matrix has been of interest in connection with the "cutoff phenomenon" of transient behavior of Markov chains [15,16,26].
} 
that the value $\lambda \in \mathbf{C}$ is not taken by $f\left(x_{*}^{-}, \mathbf{R}\right)$ or $f\left(x_{*}^{+}, \mathbf{R}\right)$, so that $I\left(f, \lambda, x_{*}^{-}\right)$ and $I\left(f, \lambda, x_{*}^{+}\right)$are defined. Suppose also that the extreme coefficients of $f\left(x_{*}^{-}, \mathbf{R}\right)$ and $f\left(x_{*}^{+}, \mathbf{R}\right)$ are nonzero in the same sense as in Theorem 1 . If $I\left(f, \lambda, x_{*}^{-}\right)>I\left(f, \lambda, x_{*}^{+}\right)$, then there exist constants $C_{1}, C_{2}>0$ and $M>1$ such that for all sufficiently large $N$ there exists a nonzero pseudoeigenmode $v^{(N)}$ with

$$
\frac{\left\|\left(A^{(N)}-\lambda\right) v^{(N)}\right\|}{\left\|v^{(N)}\right\|} \leq M^{-N}
$$

and

$$
\frac{\left|v_{j}^{(N)}\right|}{\max _{j}\left|v_{j}^{(N)}\right|} \leq C_{1} \exp \left(-C_{2} N\left|x_{j}-x_{*}\right|\right) \quad(\bmod 2 \pi) .
$$

If $I\left(f, \lambda, x_{*}^{-}\right)<I\left(f, \lambda, x_{*}^{+}\right)$, then the same conclusions hold with $A^{(N)} r e-$ placed by $A^{(N)^{T}}$.

Proof. Let us abbreviate the winding numbers by $I^{-}$on the left and $I^{+}$ on the right and assume $I^{-}>I^{+}$; the case $I^{-}<I^{+}$follows by taking the transpose. We assume $m^{-}, m^{+} \geq 0$ for simplicity. Following (39), Lemma 1 gives us spaces $\mathcal{S}_{\eta}^{\text {(right) }}$ of dimension $m^{+}-I^{+}$and $\mathcal{S}_{\eta}^{(\text {left })}$ of dimension $n^{-}+I^{-}$. Now the band structure of our variable coefficient recurrence relation is such that the solution near $x_{*}$ is determined by the $\left(m^{+}+n^{-}\right)$-dimensional linear space of values taken in the $m^{+}$points to the left and the $n^{-}$points to the right of the discontinuity. Since $m^{+}-I^{+}+n^{-}+I^{-}>m^{+}+n^{-}$, it follows that $\mathcal{S}_{\eta}^{\text {(right) }}$ and $\mathcal{S}_{\eta}^{\text {(left) }}$ must have a nonempty intersection, and this gives us our wave packet, just as in the proof of Theorem 1.

An example of a matrix with a discontinuous symbol is shown in Fig 14. Here $A$ is a $140 \times 140$ periodic tridiagonal matrix: the superdiagonal has value 2 and the subdiagonal is -1 in rows $2-71$ and +1 in rows $72-140$ and in the corner position $a_{1,140}$. Thus the symbol has a jump at $x_{*}=\pi$ :

$$
f(x, k)= \begin{cases}-\exp (\mathrm{i} k)+2 \exp (-\mathrm{i} k) & \text { for } x<x_{*}, \\ +\exp (\mathrm{i} k)+2 \exp (-\mathrm{i} k) & \text { for } x>x_{*}\end{cases}
$$

as $x$ and $k$ range over all real values, the symbol describes two ellipses but not the region interior to them. The selected values $\lambda=1.6$ lies between the two, with a jump in winding number from 0 to -1 as $x$ passes through $x_{*}$, and the figure shows the resulting localized wave packet. This matrix is a discontinuous analogue of the "target matrix" shown earlier in Fig. 10.

Although Theorem 4 covers this example, it would not apply to a similar kind of a matrix in which the discontinuity between $f\left(x_{*}^{-}, k\right)$ and $f\left(x_{*}^{+}, k\right)$ occurred over several rows, corresponding for example to a "anti-diagonal" rather than "horizontal" discontinuity in the matrix. In seeking a generalization in this direction one might expect that detailed attention to the nature of the discontinuity would be needed in order to ensure that the exponentially good wave packet pseudomodes persist. In fact, the opposite is true: they persist under arbitrary matrix alterations of any kind whatsoever, provided they are confined to a finite number of rows near the discontinuity. The following result sets forth this surprising state of affairs. This conclusion is consistent with the findings of [1] and [7] (see e.g. Fig. 4 of [7]) that 

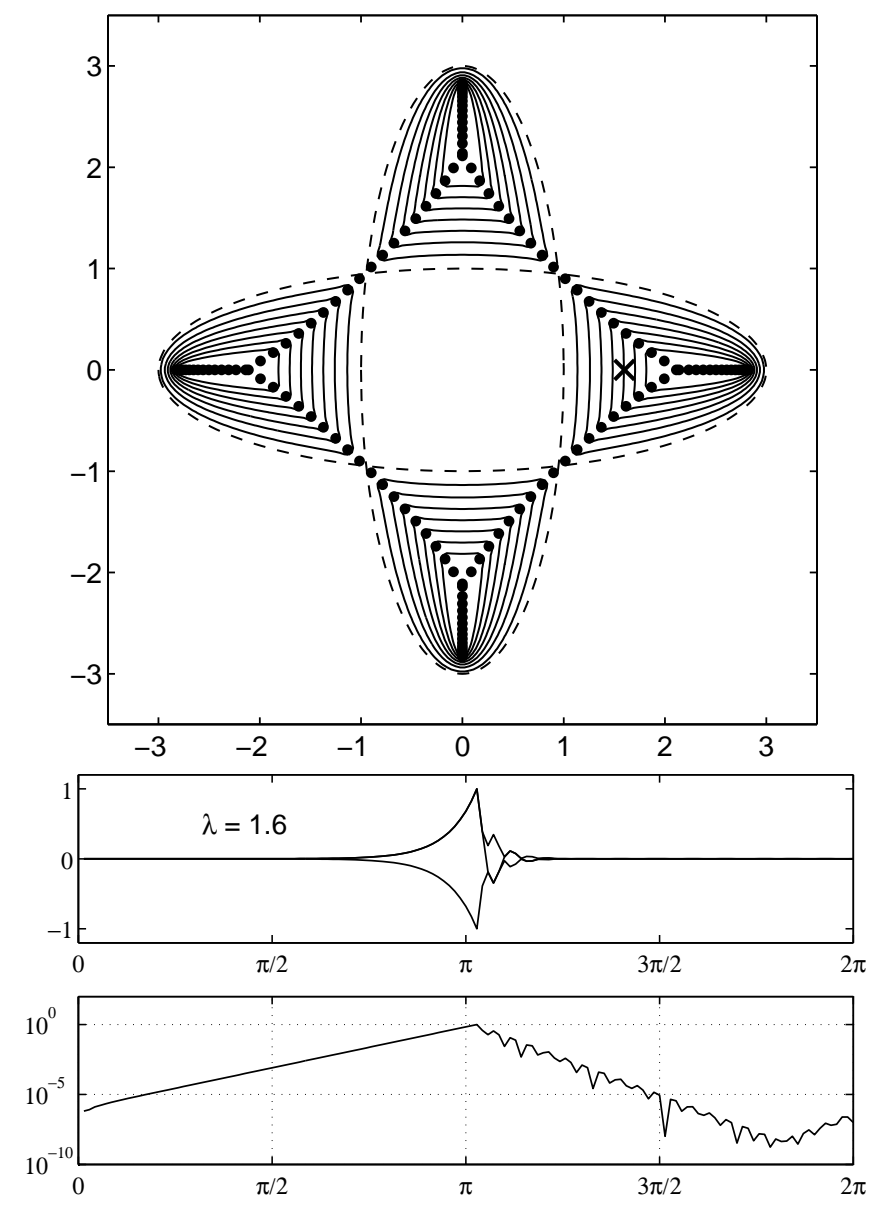

Figure 14: "Two ellipses matrix" (44) with $N=140, \varepsilon=10^{-2}, 10^{-3}, \ldots, 10^{-10}$. The $\varepsilon$-pseudoeigenvector for $\lambda=1.6$ (cross) has $x_{*}=\pi$ and $\varepsilon=8.84 \times 10^{-7}$ (shown on linear and log scales). The left and right lobes of the pseudospectra correspond to wave packet pseudomodes of $A^{(N)}$, and the top and bottom lobes to wave packet pseudomodes of $A^{(N)^{T}}$.

whereas alterations of certain entries of large nonsymmetric banded Toeplitz matrices may add anomalous eigenvalues to the spectrum, they have little effect on the pseudospectra.

Theorem 5. Let $J \geq 0$ be a fixed integer. Let $\left\{A^{(N)}\right\}$ be a family of matrices of any of the kinds described in Theorems 1-4, except that for each $N$, the $J$ rows of $A^{(N)}$ closest to $x_{*}$ are modified arbitrarily, not only inside the band but potentially in any and all positions. (In the case of Theorem 3, replace "rows" by "columns".) Then the conclusions of Theorems 1-4 still hold.

Proof. The situation is indicated schematically in Fig. 15. (We consider the wave packet case of Theorems 1-2 and 4; for Theorem 3 one takes the transpose as usual.) Our wave packet pseudomode will be a grid function that satisfies the eigenvalue equation $(A-\lambda) v=0$ exactly throughout a region $x_{*}-\Delta x<x<x_{*}+\Delta x$ (i.e., in $O(N)$ points to either side of the interface). In this region, following (39), consider the vector space of 


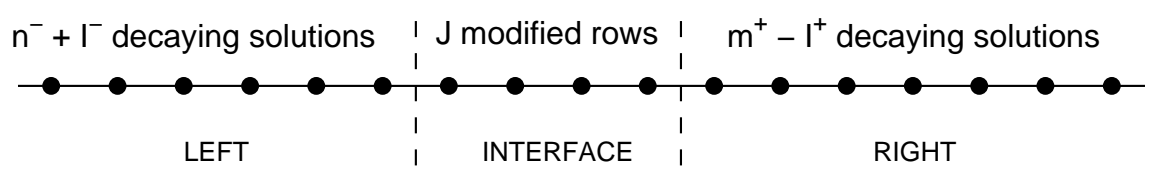

Figure 15: Setup for the proof of Theorem 5.
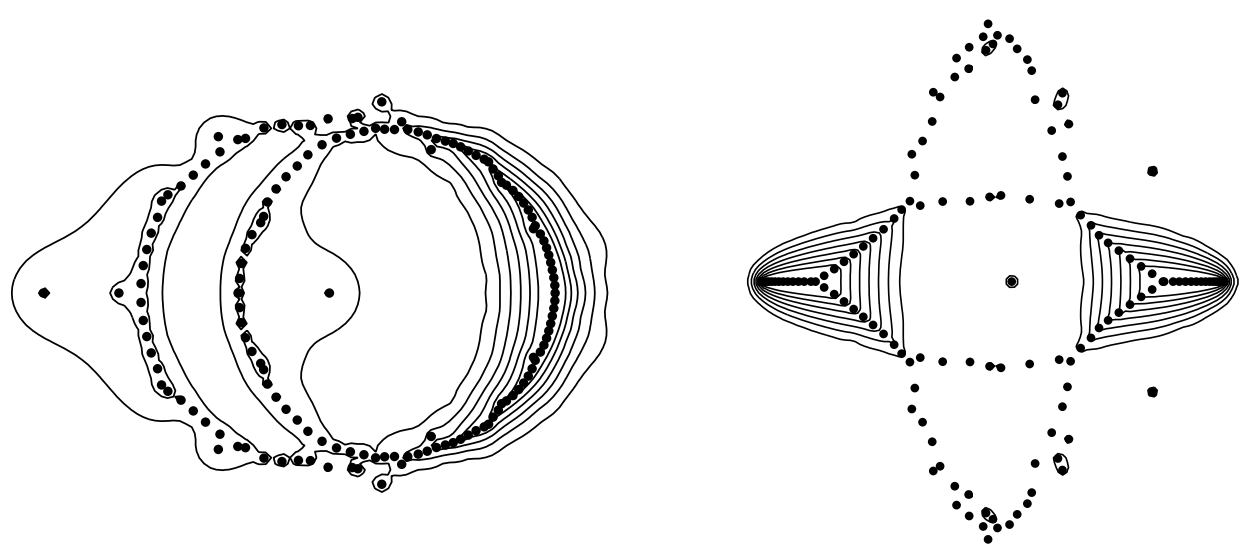

Figure 16: Repetition of Figs. 9 and 14, but now with four rows in the middle of each matrix replaced by rows of independent random numbers from the standard normal distribution. As established by Theorem 5, the lobes of each figure corresponding to wave packet pseudomodes of $A^{(N)}$ are hardly affected, while the other lobes, corresponding to wave packet pseudomodes of $A^{(N)^{T}}$, are entirely undone. These effects are reversed if columns rather than rows are altered.

dimension $\left(n^{-}+I^{-}\right)+J+\left(m^{+}-I^{+}\right)$consisting of grid functions composed of an arbitrary exponentially decaying solution to the variable coefficient recurrence relation on the left, an arbitrary exponentially decaying solution on the right, and arbitrary values in the $J$ points of the interface. Now consider what is required for such a grid function to satisfy the eigenvalue equation. At the right edge of the left region there are $n^{-}$homogeneous equations to be satisfied, and similarly there are $m^{+}$homogeneous equations to be satisfied at the left edge of the right region. In addition there are $J$ homogeneous equations of entirely arbitrary form to be satisfied in the middle region. All together, we have a linear problem involving $n^{-}+m^{+}+J$ homogeneous equations in a space of dimensional $n^{-}+m^{+}+J+\left(I^{-}-I^{+}\right)$. Since $I^{-}-I^{+}>0$ by assumption, the problem has a nonzero solution, and this is our exponentially good wave packet.

Figure 16 illustrates this striking robustness of wave packet pseudomodes.

As a corollary of Theorem 5 we may derive the main result (Theorem 3.2) of Reichel and Trefethen [34].

Corollary 1. Let $\left\{T^{(N)}\right\}$ be a family of banded Toeplitz matrices with symbol $f(k)$. Let $\lambda$ be a complex number with $f(k) \neq \lambda$ for all $k \in \mathbf{R}$ and 
let $I(f, \lambda)$ be the winding number of $f([0,2 \pi])$ about $\lambda$. If $I(f, \lambda)<0$, then there exist constants $C_{1}, C_{2}>0$ and $M>1$ such that for all sufficiently large $N$ there exists a nonzero pseudoeigenmode $v^{(N)}$ with

$$
\frac{\left\|\left(T^{(N)}-\lambda\right) v^{(N)}\right\|}{\left\|v^{(N)}\right\|} \leq M^{-N}
$$

and

$$
\frac{\left|v_{j}^{(N)}\right|}{\max _{j}\left|v_{j}^{(N)}\right|} \leq C_{1} \exp \left(-C_{2} N\left|x_{j}\right|\right) .
$$

If $I(f, \lambda)>0$, then the same conclusions hold with (46) replaced by

$$
\frac{\left|v_{j}^{(N)}\right|}{\max _{j}\left|v_{j}^{(N)}\right|} \leq C_{1} \exp \left(-C_{2} N\left|2 \pi-x_{j}\right|\right) .
$$

Proof. Consider the family of twisted Toeplitz matrices $\left\{A^{(N)}\right\}$ where for each $N, A^{(N)}$ is the $2 N \times 2 N$ matrix with $T^{(N)}$ in the upper-left $N \times N$ block and zero elsewhere. This is a twisted Toeplitz family whose symbol has discontinuities at $x_{*}=0$ and $x_{*}=\pi$, where the winding numbers change from 0 to $I(f, \lambda)$ and then back again. Theorem 5 does the rest.

\section{Double crossings and structural stability}

The reader may have noticed that we have not applied our theorems to the example of Figs. 1-4 with which we began this paper. The reason is that our theorems do not apply. For this example, as one characteristic root of the recurrence relation crosses into the unit disk, another crosses out, causing the dimensionality arguments we have used to fail. Instead, to explain this example and others like it, one must make use of the smoothness of the symbol.

Let us identify the problem algebraically. For the family of twisted Toeplitz matrices (2), the symbol is $f(x, k)=2 \sin x-2 \mathrm{i} \sin k$ and the twist ratio (35) is $\mathcal{T}(x, k)=\mathrm{i} \cos x / \cos k$. For any choice of $\lambda$ with $-2<$ $\operatorname{Re} \lambda, \operatorname{Im} \lambda<2$, there are two choices of $x_{*}$ and two choices of $k_{*}$. For example, for $\lambda=1$ as in Figs. 3-4, we have

$$
x_{*}=\sin ^{-1}\left(\frac{1}{2}\right) \approx 0.5236 \quad \text { or } \quad \pi-\sin ^{-1}\left(\frac{1}{2}\right) \approx 2.618
$$

and

$$
k_{*}=0 \quad \text { or } \pi .
$$

Now the appearance of two values of $x_{*}$ is no problem; Theorems 1-4 apply locally near any appropriate value $x_{*}$, and we have already seen in Fig. 13 an example with two values of $x_{*}$. The appearance of two values of $k_{*}$, however, is a problem indeed. Their effect will be that in Lemma 2, as one eigenvalue of the matrix $C(x)$ crosses the unit circle from outside to inside, another will cross from inside to outside. The winding number will 

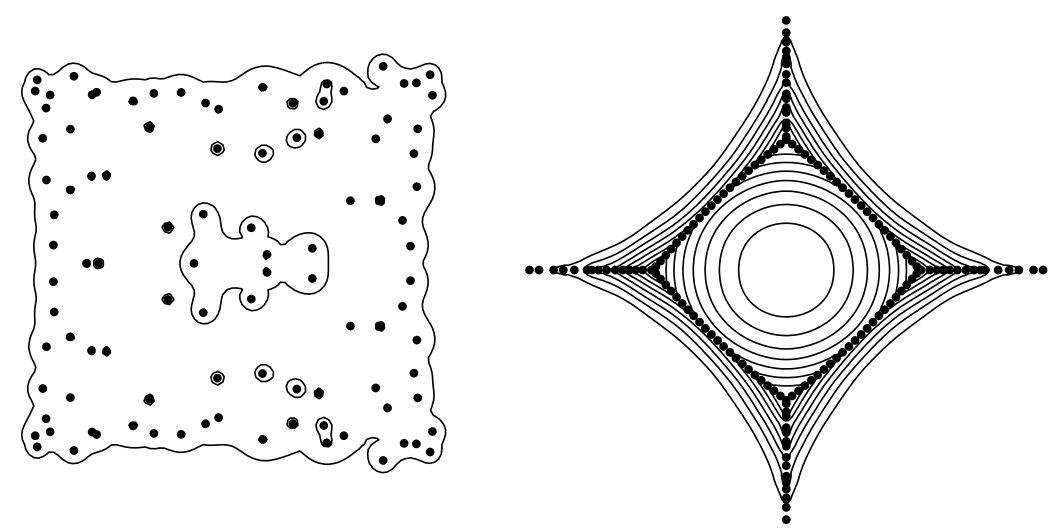

Figure 17: Repetition of Figs. 1 and 10, but now with each entry of the matrix multiplied by 1.1 or 0.9 at random. The loss of smoothness destroys the pseudospectra in the first case, where there is a double-crossing symbol, but has little effect in the second, which could be said to be structurally stable.

accordingly not change, and we will not have subspaces of solutions decaying in each direction of sufficient dimension to conclude that there must be a nonempty intersection of solutions that decay in both directions. In terms of the statement of Theorems 1-3, we have a failure of the condition that $f\left(x_{*}, k\right) \neq \lambda$ for all real $k \not \equiv k_{*}(\bmod 2 \pi)$.

The problem is not an artifact of our proofs but genuine. In general, if a family of twisted Toeplitz matrices has a symbol with a double crossing, by which we mean two or more values of $k_{*}$ for a single value of $x_{*}$ associated with some number $\lambda=f\left(x_{*}, k_{*}\right)$, then exponentially good wave packet pseudomodes need not exist for this $\lambda$. Figure 17 illustrates this numerically by repeating Figs. 1 and 10, but now with each entry of each matrix increased or decreased by $10 \%$, at random. We see that the pseudospectra of Fig. 1 are largely destroyed, while those of Fig. 10 hardly change at all. The former depend on smoothness of the symbol, and the latter do not.

To account for Figs. 1-4, one would have to go beyond the theorems of this paper. In a general sense the idea would be as follows. If a function is smooth with respect to $x$, then its transform is localized with respect to $k$. Thus it should be possible to construct good wave packets entirely from energy at wave numbers close to $k_{*}$, avoiding any other values $k_{*}^{\prime}$ at which $f\left(x_{*}, k_{*}\right)$ takes the same value $\lambda$. Borthwick and Uribe [5] present an argument of this kind that establishes the existence of rapidly (but not necessarily exponentially) decreasing pseudomodes under the assumption that the symbol is smooth.

\section{Discussion}

As mentioned in the Introduction, the phenomena we have discussed for matrices have analogues for differential and pseudodifferential operators, which have been investigated by Davies, Zworski, and others $[11,12,13,14,48,49]$. 
The analogy is close. For constant-coefficient nonselfadjoint differential operators, one finds exponentially good pseudoeigenvectors localized at a boundary [13,32], just as for nonhermitian Toeplitz matrices, whereas when the coefficients become variable, wave packet pseudoeigenvectors appear. In [14] and [48] it is shown that if the symbol is holomorphic, then the pseudoeigenvectors are exponentially or nearly exponentially good. Our theorems show that smoothness of the symbol is an unnecessary assumption in the matrix case, except in cases of a double-crossing symbol, and we believe the same is true for differential operators, as we hope to report in forthcoming work.

We hope that in the future, a greater understanding will be developed of the physical significance of wave packet pseudomodes for both matrices and differential operators. Such pseudomodes have already been studied for fluid mechanics problems related to the Orr-Sommerfeld operator by Reddy, Schmid and Henningson [33] and Chapman [10]. Davies has considered wave packet pseudomodes of complex harmonic oscillators $[11,12,13]$, and a similar complex differential operator was considered in [42]. Bender and colleagues have investigated strongly nonnormal " $\mathcal{P} \mathcal{T}$-symmetric" and

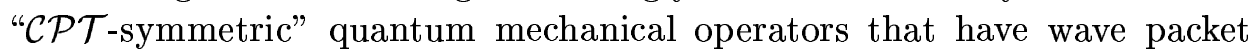
pseudomodes, though their analysis of them considers just the true eigenmodes without questioning their physical significance [2]; no doubt there are also other examples of operators with wave packet pseudomodes in the literature. In an era when a field called "nonhermitian quantum mechanics" is becoming established $[3,20]$, we expect that further applications will emerge in which the physics is determined not by eigenmodes but by wave packet pseudomodes.

\section{Acknowledgments}

Much of the background to this article lies in three years of enjoyable collaboration by LNT with Mark "Gateway" Embree and Thomas "EigTool" Wright at the Oxford University Computing Laboratory during 1999-2002; both Embree and Wright also contributed valuable suggestions to this text. We are grateful to Brian Davies (King's College London) for teaching us about wave packet pseudomodes in the continuous context, to Maciej Zworski (University of California, Berkeley) for pointing out the connection of these wave packets to the commutator condition and other topics in the theory of PDE and semiclassical mechanics, and to Alejandro Uribe (University of Michigan) for pointing out the connection to Berezin-Toeplitz operators. Besides these, a number of other colleagues have made suggestions that have improved our understanding or improved this paper, notably Carl Bender (Washington University), Albrecht Böttcher (Chemnitz), Jonathan Goodman (New York University), Michael Hitrik (University of California, Berkeley), Hinke Osinga (University of Bristol), Jim Sethna (Cornell University), Michael Taylor (University of North Carolina), and Eugene Tyrtyshnikov (Russian Academy of Sciences).

\section{References}

[1] R. M. Beam and R. F. Warming, The asymptotic spectra of banded Toeplitz and quasi-Toeplitz matrices, SIAM J. Sci. Comp. 14 (1993), 971-1006. 
[2] C. M. Bender, S. Boettcher and P. N. Meisinger, $\mathcal{P} \mathcal{T}$-symmetric quantum mechanics, J. Math. Phys. 40 (1999), 2201-2229.

[3] C. M. Bender, D. C. Brody and H. F. Jones, Complex extension of quantum mechanics, Phys. Rev. Lett. 89 (2002), ??-??.

[4] C. M. Bender and S. A. Orszag, Advanced Mathematical Methods for Scientists and Engineers, McGraw-Hill, New York, 1978.

[5] D. Borthwick and A. Uribe, On the pseudospectra of Berezin-Toeplitz operators, submitted.

[6] A. Böttcher, Pseudospectra and singular values of large convolution operators, J. Int. Eqs. Applics. 6 (1994), 267-301.

[7] A. Böttcher, M. Embree and V. I. Sokolov, The spectra of large Toeplitz band matrices with a randomly perturbed entry, Math. Comp., to appear.

[8] A. Böttcher and B. Silbermann, Introduction to Large Truncated Toeplitz Matrices, Springer-Verlag, New York, 1999.

[9] K. P. Bube and J. C. Strikwerda, Interior regularity estimates for elliptic systems of difference equations, SIAM J. Numer. Anal. 20 (1980), 653-670.

[10] S. J. Chapman, Subcritical transition in channel flows, J. Fluid Mech. 451 (2002), 35-97.

[11] E. B. Davies, Pseudo-spectra, the harmonic oscillator and complex resonances, Proc. Roy. Soc. Lond. A 455 (1999), 585-599.

[12] E. B. Davies, Semi-classical states for non-self-adjoint Schrödinger operators, Comm. Math. Phys. 200 (1999), 585-599.

[13] E. B. Davies, Pseudospectra of differential operators, J. Operator Theory 43 (2000), 243-262.

[14] N. Dencker, J. Sjöstrand, and M. Zworski, Pseudospectra of semi-classical (pseudo)differential operators, manuscript, 2002.

[15] P. Diaconis, The cutoff phenomenon in finite Markov chains, Proc. Natl. Acad. Sci. USA 93 (1996), 1659-1664.

[16] P. Diaconis, R. L. Graham and J. A. Morrison, Asymptotic analysis of a random walk on a hypercube with many dimensions, Random Struct. and Alg. 1 (1990), $51-72$.

[17] J. J. Duistermaat and J. Sjöstrand, A global construction for pseudo-differential operators with non-involutive characteristics, Inv. Math. 20 (1973), 209-225.

[18] M. Embree and L. N. Trefethen, Pseudospectra Gateway, web site http:// www. comlab.ox.ac.uk/pseudospectra.

[19] I. C. Gohberg and I. A. Fel'dman, Convolution Equations and Projection Methods for Their Solution, AMS, Providence, RI, 1974.

[20] N. Hatano and D. R. Nelson, Vortex pinning and non-Hermitian quantum mechanics, Phys. Rev. B 56 (1997), 8651-8673.

[21] N. J. Higham and P. A. Knight, Matrix powers in finite precision arithmetic, SIAM J. Matrix Anal. Applics. 16 (1995), 343-358.

[22] L. Hörmander, Differential equations without solutions, Math. Ann. 140 (1960), 169-173.

[23] L. Hörmander, On the existence and the regularity of solutions of linear pseudodifferential equations, Ens. Math. 17 (1971), 99-163.

[24] L. Hörmander, The Analysis of Linear Partial Differential Operators IV, Springer-Verlag, Berlin, 1985.

[25] M. C. Irwin, On the stable manifold theorem, Bull. Lond. Math. Soc. 2 (1970), 196-198.

[26] G. F. Jónsson and L. N. Trefethen, A numerical analyst looks at the "cutoff phenomenon" in card shuffling and other Markov chains, in D. F. Griffiths, D. J. Higham, and G. A. Watson, eds., Numerical Analysis 1997, Longman, Harlow, Essex, UK, 1998, pp. 150-178. 
[27] M. Kac, W. L. Murdock and G. Szegö, On the eigen-values of certain hermitian forms, J. Rat. Mech. Anal. 2 (1953), 767-800.

[28] H. J. Landau, On Szegö's eigenvalue distribution theory and non-Hermitian kernels, J. d'Analyse Math. 28 (1975), 335-357.

[29] H. J. Landau, The notion of approximate eigenvalues applied to an integral equation of laser theory, Quart. Appl. Math. 35 (1977), 165-172.

[30] H. Lewy, An example of a smooth linear partial differential equation without solution, Ann. Math. 66 (1957), 155-158.

[31] A. Majda, J. McDonough, and S. Osher, The Fourier method for nonsmooth initial data, Math. Comp. 32 (1978), 1041-1081.

[32] S. C. Reddy, Pseudospectra of Wiener-Hopf integral operators and constantcoefficient differential operators, J. Int. Eqs. Appl. 5 (1993), 369-403.

[33] S. C. Reddy, P. J. Schmid, and D. S. Henningson, Pseudospectra of the OrrSommerfeld operator, SIAM J. Appl. Math. 53 (1993), 15-47.

[34] L. Reichel and L. N. Trefethen, Eigenvalues and pseudo-eigenvalues of Toeplitz matrices, Lin. Alg. Applics. 162-164 (1992), 153-185.

[35] M. Shub, Global Stability of Dynamical Systems, Springer-Verlag, New York, 1987.

[36] R. P. Stevenson, On the Validity of Local Mode Analysis of Multi-Grid Methods, thesis, Utrecht, 1990.

[37] G. W. Stewart, On the powers of a matrix with perturbations, U. of Maryland Comp. Sci. report TR-4317, revised Jan. 2002.

[38] M. E. Taylor, Pseudodifferential Operators, Princeton U. Press, Princeton, 1981.

[39] P. Tilli, Locally Toeplitz sequences: spectral properties and applications, Lin. Alg. Appl. 278 (1998), 91-120.

[40] L. N. Trefethen (1992), Pseudospectra of matrices, in D. F. Griffiths and G. A. Watson, eds., Numerical Analysis 1991, Longman Scientific and Technical, Harlow, Essex, UK, pp. 234-266.

[41] L. N. Trefethen, Pseudospectra of linear operators, SIAM Review 39 (1997), 383-406.

[42] L. N. Trefethen, Computation of pseudospectra, Acta Numerica 8 (1999), 247295.

[43] E. E. Tyrtyshnikov, A unifying approach to some old and new theorems on distribution and clustering, Lin. Alg. Appl. 232 (1996), 1-43.

[44] R. Vaillancourt, On the stability of Friedrichs' scheme and the modified LaxWendroff scheme, Math. Comp. 24 (1970), 767-770.

[45] J. H. Wilkinson, The Algebraic Eigenvalue Problem, Clarendon Press, Oxford, 1965.

[46] T. G. Wright, EigTool: a graphical tool for nonsymmetric eigenproblems, http://www. comlab.ox.ac.uk/pseudospectra/eigtool/, 2002.

[47] T. G. Wright, Algorithms and Software for Pseudospectra, DPhil thesis, Oxford University Computing Laboratory, 2002.

[48] M. Zworski, A remark on a paper of E. B. Davies, Proc. AMS 129 (2001), $2955-2957$.

[49] M. Zworski, Numerical linear algebra and solvability of partial differential equations, Comm. Math. Phys., to appear. 